\title{
Optimization of Calcine Blending During Retrieval From Binsets
}

C. M. Mohr

L. O. Nelson

D. D. Taylor

June 2000

Idaho National Engineering and Environmental Laboratory Bechtel BWXT Idaho, LLC 


\title{
Optimization of Calcine Blending During Retrieval From Binsets
}

\author{
Charles M. Mohr \\ Lee O. Nelson \\ Dean D. Taylor \\ June 2000 \\ Idaho National Engineering and Environmental Laboratory \\ High-Level Waste Department \\ Idaho Falls, Idaho 83415
}

Prepared for the

U.S. Department of Energy

Assistant Secretary for Environmental Management

Under DOE Idaho Operations Office

Contract DE-AC07-99ID13727 


\section{ABSTRACT}

This report documents a study performed during advanced feasibility studies for the INTEC Technology Development Facility (ITDF). The study was commissioned to provide information about functional requirements for the ITDF related to development of equipment and procedures for retrieving radioactive calcine from binset storage at the Idaho Nuclear Technology and Engineering Center (INTEC) at the Idaho National Engineering and Environmental Laboratory (INEEL). Calcine will be retrieved prior to treating it for permanent disposal in a national repository for high level waste. The objective this study was to estimate the degree of homogenization of the calcine that might be achieved through optimized retrieval and subsequent blending. Such homogenization has the potential of reducing the costs for treatment of the calcine and for qualifying of the final waste forms for acceptance at the repository. Results from the study indicate that optimized retrieval and blending can reduce the peak concentration variations of key components $(\mathrm{Al}, \mathrm{Zr}, \mathrm{F})$ in blended batches of retrieved calcine. During un-optimized retrieval these variations are likely to be $81-138 \%$ while optimized retrieval can reduce them to the $5-10 \%$ range. 


\section{SUMMARY}

The 1995 Settlement Agreement between the U.S. Department of Energy (DOE) and the state of Idaho mandates treatment of high level radioactive waste calcine currently stored at the Idaho Nuclear Technology and Engineering Center (INTEC) at the Idaho National Engineering and Environmental Laboratory (INEEL). Any of the proposed processes for treating this waste will require a substantial effort aimed at characterizing the calcine and developing and qualifying the processes to be employed in treating it for disposal in a national repository. In light of this requirement feasibility studies are being performed for a new facility (the INTEC Technology Development Facility, or ITDF) at INTEC to house the needed development activities. One of the activities this facility must accommodate is a system to retrieve calcine from the current storage facilities (binsets) prior to treatment. As a first step in scoping the associated testing requirements a study was performed to assess the need for optimizing the calcine retrieval system. This report documents that study.

All proposed treatment processes for calcine could be simplified and made less costly if the calcine fed to the treatment process is homogeneous in composition. Given this fact, the principal objective of this study was to obtain quantitative estimates of the degree of homogenization that could be achieved (in principal) during calcine retrieval and blending prior to treatment. The study addressed this question by hypothesizing a fully-optimal retrieval system, subject to only the following constraints: (1) The calcine is stored in the binsets in layers of differing, but known, composition, and (2) The calcine must be retrieved from each bin from the top down. Computer simulations of the hypothetical retrieval system showed that, if fully optimized, the system could indeed achieve a high degree of homogeneity in blended calcine fed to the treatment process. Once this was established, a second major study objective was formulated. This objective was to obtain a quantitative estimate of the degree of homogenization that could be achieved with practical retrieval systems which were far less complex and costly than the hypothetical system posited in addressing the first objective.

Radioactive calcine in storage at INTEC was produced by calcination of radioactive raffinates from nuclear fuel reprocessing activities at the INEEL. Calcination began in 1963 and continued 37 years, producing roughly $4,200 \mathrm{~m}^{3}$ of calcine. Principal components of the calcine include aluminum, zirconium, and fluorine, as well as other species. Because of the manner in which it was produced, batch-by-batch from raffinates of varying composition, the calcine in storage is non-homogeneous, and resides in layers of varying composition in 43 different stainless steel bins. Because of natural mixing processes that occurred during the making and transport of the calcine, the layers are not perfectly segregated in the binsets and the calcine composition varies continuously across mixing layers between different calcines. The bins containing the calcine are arranged in clusters, each cluster being housed in a concrete-shielded vault called a binset.

Retrieval of the calcine from the bins prior to treatment will entail construction of remotelyoperated pneumatic systems atop each binset. Each binset must be penetrated from the top in order to access the bins within it. Each bin must then be penetrated with a retrieval nozzle which includes a blower to fluidize calcine in the bin and a suction nozzle to draw the suspended calcine into a pneumatic transport line. The transport lines will be shielded and will convey the calcine to a blending bin for homogenization prior to treatment. Prior studies on calcine retrieval have been performed and suggest that practical systems will likely incorporate either two retrieval nozzles which are moved from binset to binset and from bin to bin, or one retrieval nozzle for each binset which is moved from bin to bin within that binset. In contrast to these systems is a system employing a separate retrieval nozzle in each of the 43 bins. The latter system was hypothesized in addressing the first objective above, and the former systems were employed in addressing the second objective. 
To obtain quantitiative estimates of the degree of homogenization achievable with the three retrieval systems described above a computerized Monte Carlo simulation of the retrieval process was employed. This simulation utilized calcine composition data compiled from process knowledge over the years. With this information, different retrieval sequences were carried out computationally, and the resulting variation of composition of the blended calcine from the homogenizing bin was calculated. Based on information obtained from vitrification personnel it was decided to characterize composition variations using the sum of squares of the variations of $\mathrm{Al}, \mathrm{Zr}$, and $\mathrm{F}$ from their average concentrations over all 4,200 $\mathrm{m}^{3}$ of stored calcine. The Monte Carlo model was used to search for the "best" retrieval sequence in the sense of minimizing composition variations of the blended, retrieved calcine. In performing this search it was hypothesized that calcine would be retrieved in $1-\mathrm{m}^{3}$ parcels from the bins, placed in a $10-\mathrm{m}^{3}$ calcine mixer, and then fully homogenized. The objective function used in the Monte Carlo search was the largest least-squares variation over all 441 batches (each $10 \mathrm{~m}^{3}$ in volume) of blended calcine produced.

Monte Carlo simulations were performed for each of the three retrieval systems described above. The resulting composition variations were then compared with those which were obtained in an earlier retrieval study which assumed a non-optimized retrieval sequence. Two sets of numbers were compared, the first being the relative standard deviations (RSDs) for $\mathrm{Al}, \mathrm{Zr}$, and $\mathrm{F}$ $[\mathrm{RSD}=($ std dev $) /($ mean value $)]$. The second set of numbers consists of the maximum relative deviations (MRDs) for the same elements $\left[M R D=\right.$ max over all batches $\left\{\left|\mathrm{c}_{\mathrm{i}}-\mathrm{c}_{\text {avg }}\right| \mathrm{c}_{\text {avg }}\right\}$, where $\mathrm{c}_{\mathrm{i}}=$ concentration of specie in question in $\mathrm{i}^{\text {th }}$ batch, "avg" = average over all calcines]. The results of the comparison are shown in the table below.

\begin{tabular}{|l|c|c|c|c|c|c|}
\hline Parameter $(\rightarrow)$ & \multicolumn{3}{|c|}{ RSD } & \multicolumn{3}{c|}{ MRD } \\
\hline Retrieval Scenario $(\downarrow)$ & Al & Zr & F & Al & Zr & F \\
\hline Non-optimized retrieval & $46 \%$ & $38 \%$ & $34 \%$ & $138 \%$ & $81 \%$ & $84 \%$ \\
\hline $\begin{array}{l}\text { Optimized retrieval } \\
(43 \text { retrieval nozzles) }\end{array}$ & $2 \%$ & $3 \%$ & $3 \%$ & $13 \%$ & $14 \%$ & $17 \%$ \\
\hline $\begin{array}{l}\text { Optimized retrieval } \\
(6 \text { retrieval nozzles) }\end{array}$ & $1 \%$ & $2 \%$ & $2 \%$ & $5 \%$ & $7 \%$ & $10 \%$ \\
\hline $\begin{array}{l}\text { Optimized retrieval } \\
(2 \text { retrieval nozzles) }\end{array}$ & $17 \%$ & $15 \%$ & $12 \%$ & $51 \%$ & $59 \%$ & $57 \%$ \\
\hline
\end{tabular}

The results of the study, summarized in the table, indicate that a significant improvement in blended calcine homogeneity may be derived from optimizing the retrieval process. In particular, the 6-retrieval nozzle system can be optimized to reduce the peak composition variation by roughly a factor of ten. Such homogenization has the potential of reducing costs for treatment of the calcine and for qualifying of the final waste forms for acceptance at a repository. In addition to this major conclusion, the following conclusions and recommendations were obtained.

\section{$\underline{\text { Conclusions }}$}

1. Optimized retrieval and blending may homogenize calcine sufficiently to justify a single flowsheet for calcine treatment.

2. A retrieval system required to achieve fully optimized calcine retrieval is probably prohibitively costly and complex. 
3. A partially optimized retrieval system employing one retrieval line for every binset can reduce the composition variation sufficiently to warrant its further consideration.

4. A minimum-cost retrieval system, employing only two suction lines, can reduce composition variations by a factor of 2-3 from those with a non-optimized system.

5. Mixing between calcine strata in the bins does not appear to affect the homogeneity achievable in blended calcine batches.

\section{$\underline{\text { Recommendations }}$}

1. Direct efforts at defining a rationale and a strategy for sampling calcine in the binsets. (One possible strategy has been suggested in Section 4.3 of this report.)

2. Perform optimized retrieval studies on the effect of uncertainty in the locations of the interfaces between calcine strata in the bins.

3. Develop real-time assay techniques for use during retrieval to identify interfaces between calcine types. Such techniques would be required for optimized retrieval.

4. Perform additional retrieval simulations to further improve the homogeneity of blended, retrieved calcine.

5. Plan future glass composition variation studies (CVSs) to reflect the expected calcine feed variations as described in this report.

6. Perform a tradeoff study to assess the merit (from a cost standpoint) of optimizing the retrieval system vs qualifying multiple flowsheets and/or final waste forms to accommodate large variations in calcine feed composition.

7. Review ITDF functional requirements and design to account for the specifics of likely approaches to sampling, retrieving and blending calcine.

8. Extract a limited number of random calcine samples from the binset to qualify the database used for retrieval optimization.

9. Investigate the upper practical limit on the size of a calcine blending bin for retrieved calcine. 


\section{CONTRIBUTORS}

David B. Lord

Byron L. Blakely

Charles M. Mohr

Lee O. Nelson

Dean D. Taylor
Project Management

Technical Lead

Chemical \& Process Engineering

Chemical \& Process Engineering

Chemical \& Process Engineering 


\section{CONTENTS}

ABSTRACT

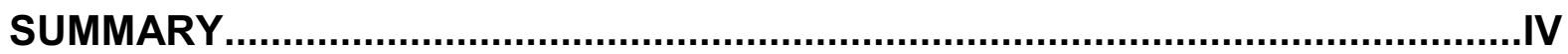

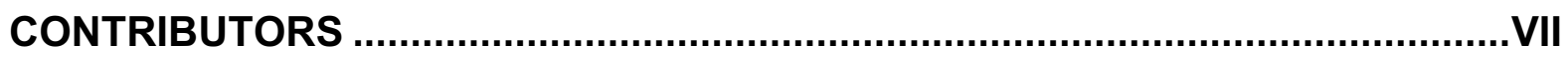

ACRONYMS \& ABBREVIATIONS

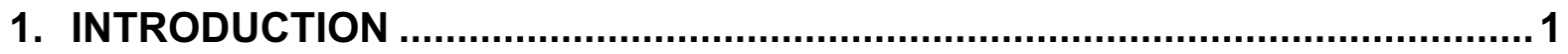

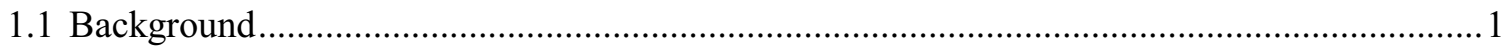

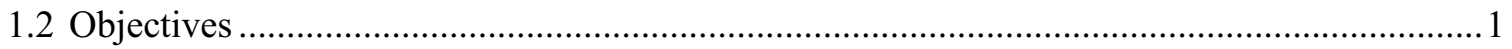

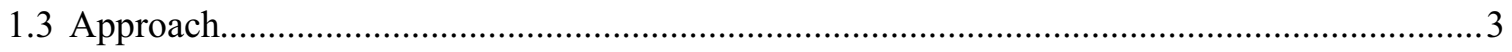

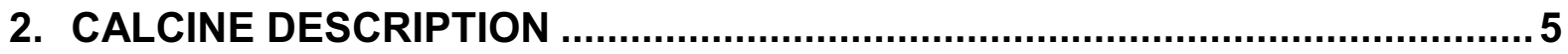

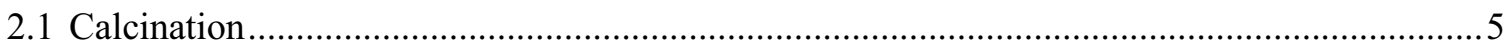

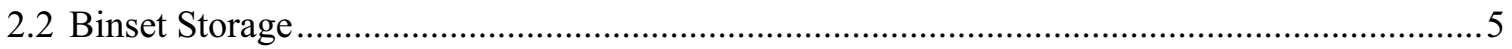

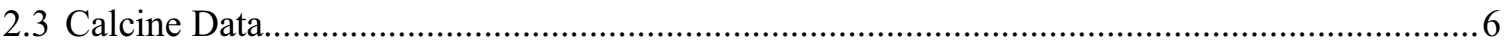

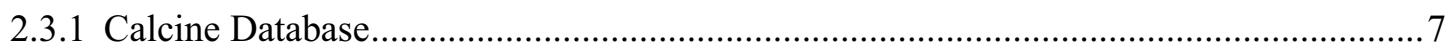

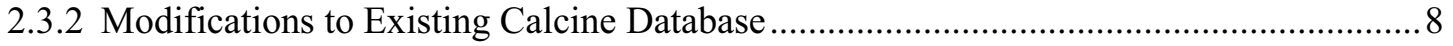

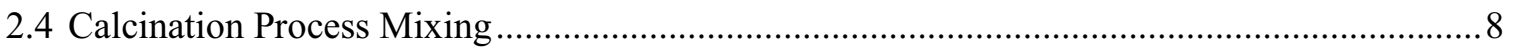

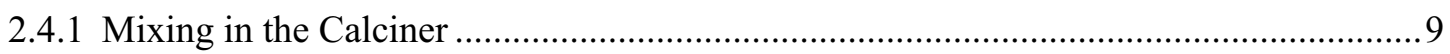

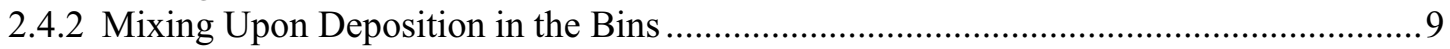

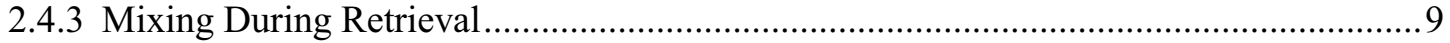

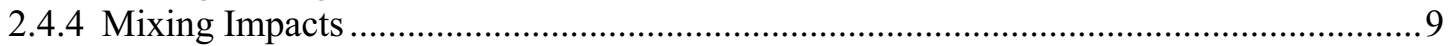

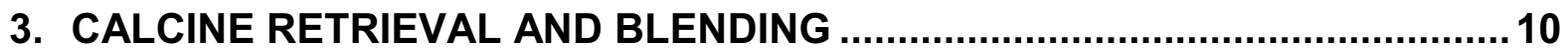

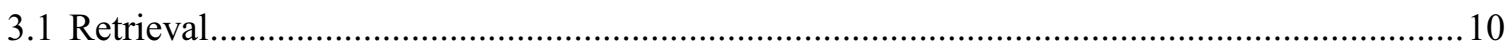

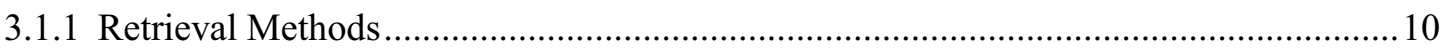

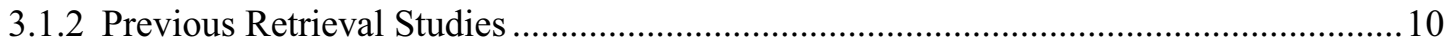

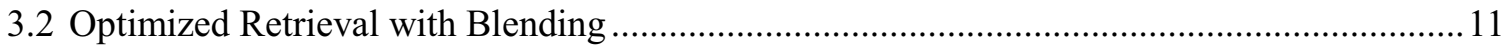

4. RETRIEVAL OPTIMIZATION STUDIES ..................................................... 14

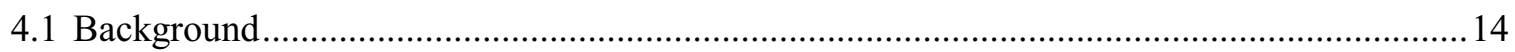

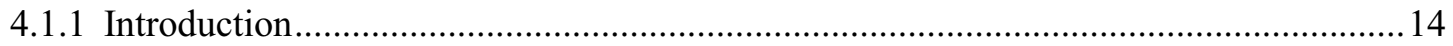

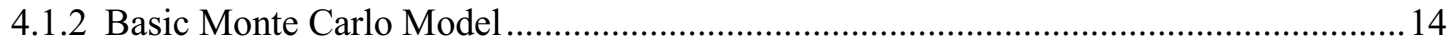

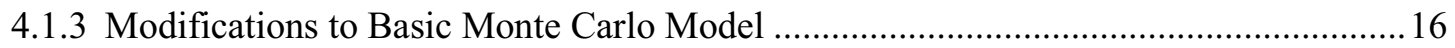

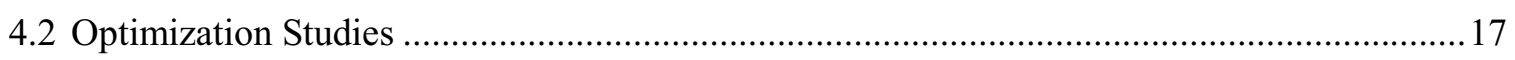

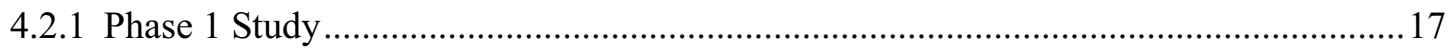

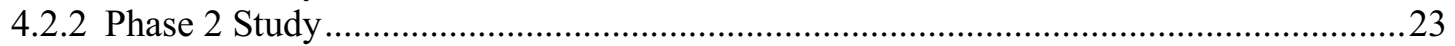

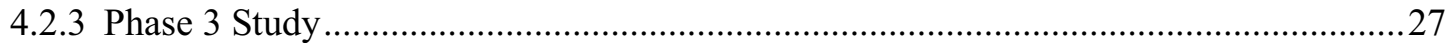

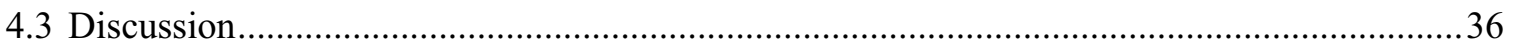

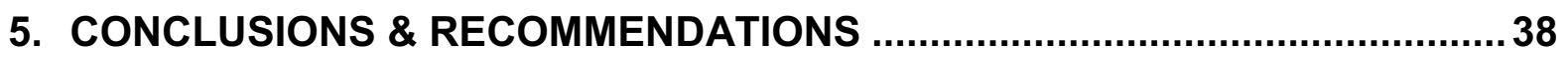

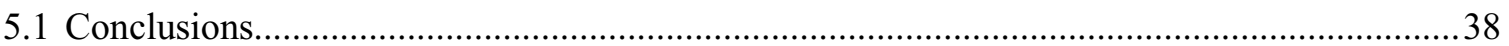




\section{REFERENCES}

\section{FIGURES}

Figure 1-1: Estimated calcine composition variation from first retrieval study. ................................

Figure 4-1: Batch-by-batch composition variation (major species, Phase 1 study)............................2 21

Figure 4-2: Batch-by-batch composition variation (major species, Phase 1 study)...........................22

Figure 4-3: Batch-by-batch composition variation (major species, Phase 1 study)............................25

Figure 4-4: Batch-by-batch composition variation (major species, Phase 1 study)...........................26

Figure 4-5: Batch-by-batch composition variation (major species, Phase 3 study-Case A).............30

Figure 4-6: Batch-by-batch composition variation (major species, Phase 3 study-Case A)............31

Figure 4-7: Batch-by-batch composition variation (major species, Phase 3 study-Case B)............33

Figure 4-8: Batch-by-batch composition variation (major species, Phase 3 study-Case C) .............35

\section{TABLES}

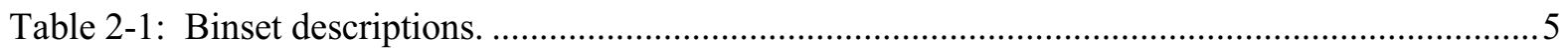

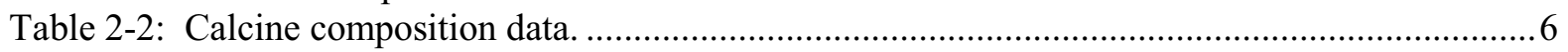

Table 4-1: Statistics of composition variation of optimized species (Phase 1 study)......................... 19

Table 4-2: Statistics of composition variation of other species (Phase 1 study)................................ 19

Table 4-3: Statistics of composition variation of Al, Zr, and $\mathrm{F}$ for non-optimized retrieval............... 19

Table 4-4: Statistics of composition variation of optimized species (Phase 2 study)........................23

Table 4-5: Statistics of composition variation of other species (Phase 2 study).................................24

Table 4-6: Statistics of composition variation of optimized species (Phase 3 study - Case A).........28

Table 4-7: Statistics of composition variation of other species (Phase 3 study - Case A)................28

Table 4-8: Statistics of composition variation of optimized species (Phase 3 study-Case A, with

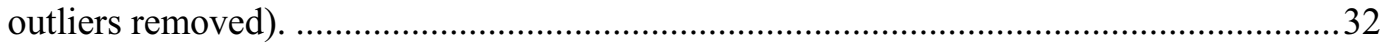

Table 4-9: Statistics of composition variation of optimized species (Phase 3 study - Case B)........... 32

Table 4-10: Statistics of composition variation of optimized species (Phase 3 study - Case C).........34 


\section{ACRONYMS \& ABBREVIATIONS}

CSTR

DOE

FS

FY

INEEL

INTEC

ITDF

MRD

RSD

SD

VB
Continuously Stirred Tank Reactor

United States Department of Energy

Feasibility Study

Fiscal Year

Idaho National Engineering and Environmental Laboratory

Idaho Nuclear Technology and Engineering Center (formerly Idaho Chemical Processing Plant, ICPP)

INTEC Technology Development Facility

Maximum Relative Deviation

Relative Standard Deviation

Standard Deviation

Visual Basic 


\section{OPTIMIZATION OF CALCINE BLENDING DURING RETRIEVAL FROM BINSETS}

\section{INTRODUCTION}

\section{$1.1 \quad$ Background}

In 1995 a Settlement Agreement was reached between the U.S. Department of Energy (DOE) and the State of Idaho dealing with treatment and disposal of radioactive wastes at the Idaho National Engineering and Environmental Laboratory (INEEL). One of the requirements in this agreement is that high level radioactive waste calcine at the INEEL be treated and made road ready by 2035 . This calcine is currently stored in stainless steel bins inside concrete vaults (hereafter these vaults are referred to as binsets) at the Idaho Nuclear Technology and Engineering Center (INTEC) at the INEEL. Tentative plans call for DOE to retrieve the calcine from the binsets and treat it as necessary, either at a facility to be constructed at the INEEL or at an offsite facility. In either case a facility must be constructed to extract calcine from the binsets and transport it to a handling facility (for treatment or packaging for offsite transport).

The options currently being considered to treat the calcine include direct cementation, vitrification and hot isostatic pressing. In addition, there are options to dissolve the calcine and separate the high and low activity components so as to reduce the total volume of the final high activity waste form. These separation options also produce cemented and/or vitrified final waste forms. For all treatment options being considered processing of the waste is made simpler and less costly by homogenizing the calcine prior to the start of processing. In addition the costs and complexity to develop treatment processes are reduced if variations in the waste feed stream composition are minimized.

In FY'99 a feasibility study (FS) examined facility requirements for development of a process to treat calcine. In FY'00 this FS was expanded to define in more detail the testing and development activities that would likely be conducted prior to implementation of the activities mandated by the 1995 Settlement Agreement. As noted above, the extent of such development activities depends on the homogeneity of the calcine fed to the process. Therefore, as part of the referenced FY'00 FS a separate study was performed to estimate the degree of homogeneity that might be achievable in the calcine fed to the treatment process.

A number of methods have been proposed to homogenize retrieved calcine prior to processing. One such method is to blend calcine as it is retrieved from the bins. This method would exploit the distribution of the calcine throughout multiple bins and binsets by retrieving calcine from two or more bins into a pre-treatment blending bin in a way that minimizes the variation in the average composition of the mixture. The study described in this report was commissioned to examine this possibility. Specifically, the study was directed at determining the degree of homogeneity that might be achieved by optimizing the selection and sequencing of retrieval points in the binsets.

\subsection{Objectives}

An early study of the possible variation in calcine composition as it is retrieved from the bins was performed by C.M. Barnes. Barnes assumed that calcine could be drawn simultaneously from 
two binsets and blended in 9,000 kg batches. Binsets were paired on the basis of their overall composition so that the average aluminum content over two binsets in a pair was as near the global average as possible. No attempt was made to optimize the sequencing of bins within a binset in retrieving the calcine beyond this initial pairing of the binsets. Thus, this study provided a baseline for further optimization of the retrieval scheme. The results of the study are shown in Figure 1-1, where the mass fractions (as \%'s) are shown for Al, Zr, and F in all the 9,000 kg calcine batches made in emptying the binsets. The figure shows a variation of roughly $6-40 \%$ in $\mathrm{Al}, 3-18 \%$ in $\mathrm{Zr}$, and 2 $25 \%$ in F. With these results representing the baseline calcine composition variation that would be likely with minimal optimization, the first objective we set out to accomplish in this study was as follows:

- Objective 1. Estimate the lowest variation in calcine composition that can be achieved through optimization of the retrieval system alone, with only one physical constraint on the retrieval system - that calcine must be drawn from each bin within the binsets from the top down. (i.e., retrieval of calcine from below the upper surface of the calcine at any time was not allowed.)

In pursuing this objective it was assumed that calcine would be retrieved from the binsets into a blending bin of a prescribed size (generally $10 \mathrm{~m}^{3}$ throughout the present study). The calcine within this blending bin was then assumed to be fully homogenized through some mechanical means. Thus, the above objective amounted to minimizing the batch-to-batch composition variations in the blending bin. This was accomplished during Phase 1 of the study. After this was done it became apparent that additional physical constraints should be placed on the retrieval system in order that it be constructable and operable at a reasonable cost. Thus, a second objective was formulated as follows:

- Objective 2. Estimate the lowest variation in calcine composition that can be achieved through optimization of the retrieval system alone, with additional physical constraints on the retrieval system which reduce its cost and complexity.

The second objective was addressed during Phases 2 and 3 of the study. 
First Study Of Blended Calcine Composition Variation

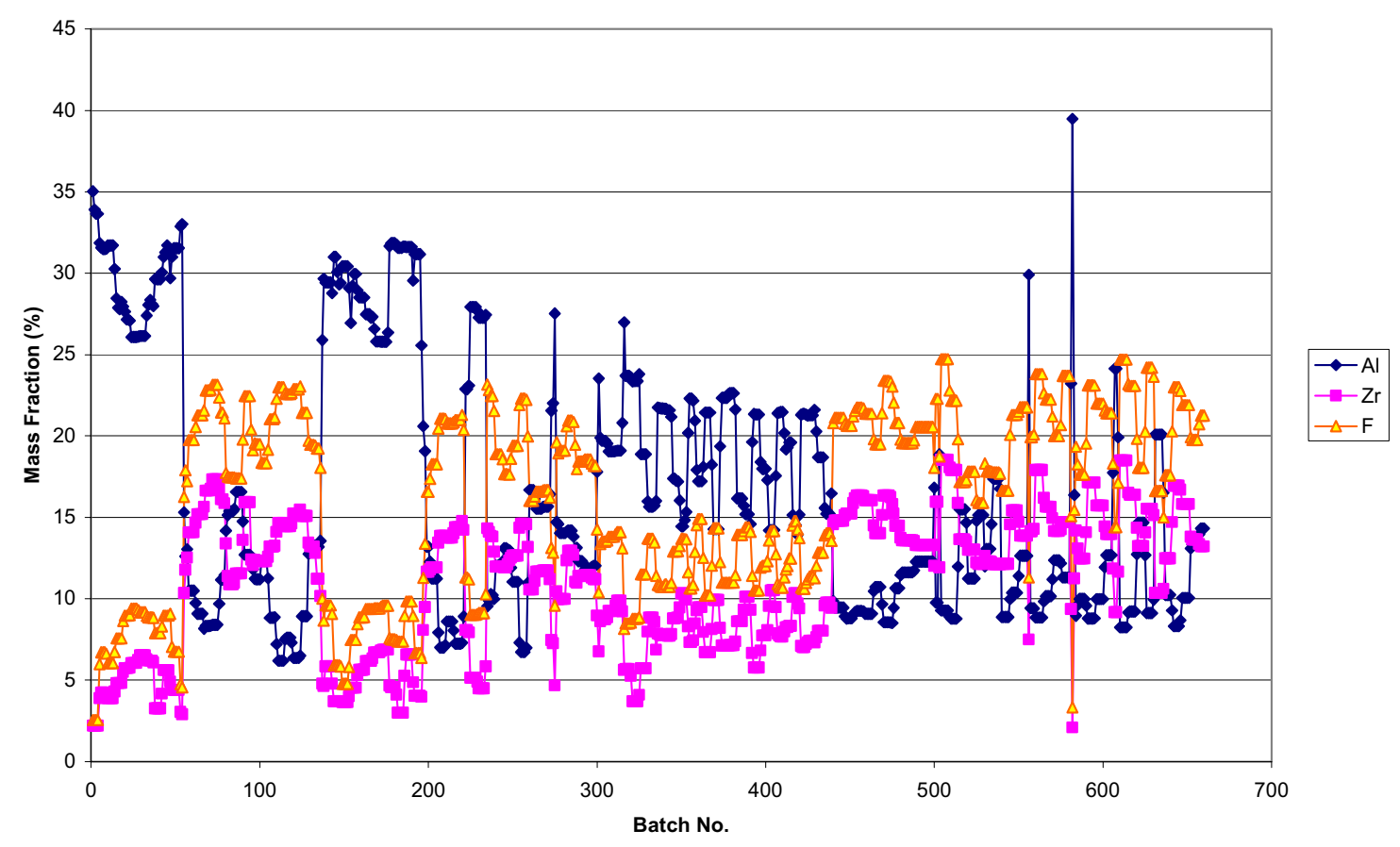

Figure 1-1: Estimated calcine composition variation from first retrieval study.

\subsection{Approach}

We achieved the above objectives by performing the following tasks:

- We obtained available data about the calcine in the binsets - compositions, layering, quality of available data, basis of available data, etc. Output from this task is discussed in Section 2.

- We reviewed work done to date on retrieval methods to get an idea of what might be feasible from the standpoint of blending. We identified possible retrieval configurations and their associated implied constraints on blending. Output from this task is discussed in Section 2.4.

- We defined the objective that an "ideal" retrieval system might be expected to accomplish with respect to blending the different calcines in the binsets prior to treatment. Then we quantified an objective function to use in optimizing the retrieval system toward the desired objective. This task is discussed in Sections 3.2, 4.1.2, 4.2.1.1, and 4.2.3.1

- We defined the "best" retrieval system that could physically be realized, if cost, operational complexity, and worker safety were not issues. 
- Using a Monte Carlo scheme we optimized calcine retrieval with the system. This scheme looked at as many retrieval/blending sequences as possible which could physically be realized and picked the one which gives the best value of the optimization function. The scheme we used is described in Sections 4.1.2 and 4.1.3. The optimization functions used are discussed in Sections 4.2.1.1 and 4.2.3.1.

- After the above optimization showed that a "best possible" retrieval system could indeed deliver significant benefits relative to the "ideal" retrieval system, we then set out to determine the degree to which such benefits might be realized with successively simpler (and less-costly) retrieval systems. The output from this task is discussed in Sections 3.2, 4.2.2, and 4.2.3 


\section{CALCINE DESCRIPTION}

\subsection{Calcination}

Nuclear fuel reprocessing activities conducted at the Idaho Nuclear Technology and Engineering Center (INTEC) have generated of over seven million gallons of acidic aqueous waste. The aqueous waste consists of a mixture of nitrates, fluorides, and dissolved spent nuclear fuel cladding. Beginning in 1963, a fluidized-bed calciner has been used to convert this aqueous waste into a more stable granular material called calcine. During 37 years of operation, $4,200 \mathrm{~m}^{3}$ of calcine have been generated from approximately $29,600 \mathrm{~m}^{3}$ of acidic liquid waste. The calcine is stored inside six concrete vaults, known as binsets.

Three major liquid wastes have been generated at INTEC: aluminum, zirconium, and sodiumbearing waste. Aluminum and zirconium waste results from nuclear fuel reprocessing activities. Sodium-bearing waste results from decontamination operations. The aqueous waste is stored in liquid waste storage tanks awaiting calcination. These liquid waste tanks have been in operation since waste-processing activities began. The composition and volume of the waste in these tanks has been monitored to support calcination activities and to maintain a process knowledge database.

Calcination occurs as aqueous waste is injected into a fluidized bed at a temperature of $400^{\circ}$ to $600^{\circ} \mathrm{C}$. As the aqueous waste is heated in the calciner, three types of changes occur: water is vaporized, nitrates decompose, and the remaining waste components are converted to a mixture of oxides, fluorides, and nitrates. Two major classifications of calcine are produced in the calciner: "product" is defined as particles with a diameter of 200 to $500 \mu \mathrm{m}$ and "fines" are defined as particles with a diameter of 10 to $200 \mu \mathrm{m}$. Calcine product and fines are transported from the calciner to storage bins by means of pneumatic conveying equipment. A cyclone above each binset is used to separate calcine from transport air. The solids fall by gravity into the bins and the dust laden transport air is returned to the calciner.

\subsection{Binset Storage}

Six binsets have been constructed on an as-needed basis to store calcine. Each binset contains a number of specially designed stainless steel bins which contain the calcine. There are a total of 43 stainless steel bins in the six binsets. Each binset is unique and has been designed to incorporate lessons learned during previous operations experience. Bin design data and calcine storage volumes for each binset are shown in Table 2-1. Binset 1 was built without any provision for future calcine retrieval. However, the remaining binsets were built to accommodate future calcine retrieval. In order to allow estimation of the bin filling height during calciner operation, thermocouples were placed at various heights within each bin. As the temperature measured by each thermocouple increased, the bin was assumed to be filled to that level.

Table 2-1: Binset descriptions.

\begin{tabular}{|c|c|c|l|l|}
\hline $\begin{array}{c}\text { Bin } \\
\text { set }\end{array}$ & $\begin{array}{c}\text { Usable Storage } \\
\text { Volume }\left(\mathrm{m}^{3}\right)\end{array}$ & \% Full & Bin Configuration & Dimensions \\
\hline 1 & 227 & 95.7 & $\begin{array}{l}4 \text { sets of 3 annular bins } \\
(12 \text { bins total })\end{array}$ & $\begin{array}{c}25 \text {-ft tall } \\
\text { innermost: 3-ft OD } \\
\text { middle: } 41-\text { in ID - 90-in OD }\end{array}$ \\
\hline
\end{tabular}




\begin{tabular}{|c|c|c|l|l|} 
& & & \multicolumn{1}{|c|}{ outer: 94-in ID - 142-in OD } \\
\hline 2 & 855 & 100.0 & 7 bins & 42.3-ft tall x 12-ft OD \\
\hline 3 & 1,096 & 99.5 & 7 bins & 53-ft tall x 12-ft OD \\
\hline 4 & 488 & 100.0 & 3 bins & 55-ft tall x 12-ft OD \\
\hline 5 & 992 & 100.0 & $\begin{array}{l}7 \text { bins with inner annular } \\
\text { space }\end{array}$ & 50-ft tall x 4-ft ID - 12-ft OD \\
\hline 6 & 1,506 & 42.6 & $\begin{array}{l}7 \text { bins with inner annular } \\
\text { space }\end{array}$ & 67.6-ft tall x 5-ft ID - 13.5-ft OD \\
\hline
\end{tabular}

\subsection{Calcine Data}

Calcine stored at INTEC may be classified into four major types: aluminum, zirconium, fluorinel, and sodium bearing. The average composition of each calcine type is shown in Table 2-2. The calcine solids are stratified in layers. Binset 1 and 2 contain only one or two calcine types. However, binset $3,4,5$, and 6 contain up to 50 stratified layers. The compositions shown in Table 2-2 are averages. The actual calcine composition is variable. For example, the aluminum oxide mass fraction in aluminum calcine varies from 78 to $95 \%$.

Table 2-2: Calcine composition data.

\begin{tabular}{|c|c|c|c|c|}
\hline Component & $\begin{array}{c}\text { Aluminum } \\
\text { Calcine }(w t \%)\end{array}$ & $\begin{array}{c}\text { Zirconium } \\
\text { Calcine }(w t \%)\end{array}$ & $\begin{array}{c}\text { Fluorinel Calcine } \\
(w t \%)\end{array}$ & $\begin{array}{c}\text { Sodium Bearing } \\
\text { Calcine (wt\%) }\end{array}$ \\
\hline Aluminum & 87.3 & 11.8 & 6.5 & 53.4 \\
\hline Sodium & 1.4 & 0.1 & 8.1 & 11.4 \\
\hline Nitrate & 2.6 & 1.1 & 1.0 & 23.0 \\
\hline Boron & 0.4 & 37.7 & 31.9 & 0.8 \\
\hline Calcium & & 0.3 & 0.2 & 4.0 \\
\hline Iron & 0.1 & 30.1 & 21.9 & 0.4 \\
\hline Fluoride & & & & 1.0 \\
\hline Mercury & 5.5 & 0.1 & 0.9 & 0.003 \\
\hline Potassium & 0.1 & 18.2 & 15.5 & 1.4 \\
\hline Zirconium & & & 3.7 & 0.7 \\
\hline Sulfate & 1.5 & & & 0.4 \\
\hline Phosphate & 1.1 & 0.3 & 0.1 & 0.1 \\
\hline Chloride & & & 0.1 & \\
\hline Tin & & & & \\
\hline Chromium & & & & \\
\hline
\end{tabular}




\begin{tabular}{|l|l|l|l|l|}
\hline Cadmium & & & 5.5 & 0.2 \\
\hline
\end{tabular}

\subsubsection{Calcine Database}

The database ${ }^{1}$ used to complete the calculations described in Section 3.2 consists of 315 distinct sets of information, each of which represents a layer of calcine in a bin. The database provides the calcine mass and average composition that are located between two thermocouples. Each of the 315 information sets contains the following information:

- calcine storage bin

- thermocouple number

- volume of layer

- highest recorded temperature

- calciner run number

- calciner liquid feed batch number

- mass of each chemical component (excluding oxygen).

A brief description of how the information was compiled is given in the following sections.

\subsubsection{Calcine storage bin}

The calcine storage bin was noted during calciner operations. As a result, no assumptions were required to verify this data point.

\subsubsection{Thermocouple number}

The thermocouple number was estimated from data collected during calciner operation. The thermocouple readings in each bin were taken intermittently. As a result, the exact time a particular thermocouple was covered by calcine is not available, but it can be estimated. Thermocouple data are available for the entire filling history of each bin. The day a particular thermocouple was covered is estimated to be a linear function of time. For example, the day a particular bin was completely filled is known, and the number of days the calciner was operated is known. The thermocouples within each bin were assumed to be covered over as a linear function of time.

\subsubsection{Volume of Layer}

The volume contained in each layer of calcine is calculated from the distance between each thermocouple and the diameter of the bin. The summation of these individual layers corresponds with the known bin volume.

\subsubsection{Highest recorded temperature}

The highest recorded temperature in each bin corresponds with the maximum reading recorded for each bin. This temperature is assumed to be fairly accurate as the bin temperature did not change rapidly once it was filled with calcine. 


\subsubsection{Calciner run number and liquid feed batch number}

The calciner run number and liquid feed batch number were used to obtain liquid waste composition and volume. This information was used to identify the concentration of each component present in the liquid feed and to estimate the quantity of each component present in the particular layer.

\subsubsection{Mass of each chemical component}

The mass of each chemical component was estimated from the liquid feed data and the volume of liquid processed. For example, based on thermocouple data, a particular layer was filled during a given 5 days. The masses were estimated from the molarity of each component in solution and the volume of liquid waste processed in the calciner during the period of interest. Calciner feed volume data were not available prior to 1968. Tank depletion data were used to estimate the feed volume during calciner operations of 1964 to 1968 . Feed volume data were used for estimating the mass of each component in the calcine produced after 1968.

\subsubsection{Modifications to Existing Calcine Database}

The volume and composition data presented in the calcine database were assumed to be accurate. The mass data presented in the database conflicted with the known volume and density of each layer. As a result, the volume data were used to estimate the total mass of calcine in each layer. The density of calcine was assumed to be $1.1 \mathrm{~g} / \mathrm{cc}$ for alumina calcine, $1.52 \mathrm{~g} / \mathrm{cc}$ for zirconia and fluorinel calcine, and 1.34 for sodium bearing calcine ${ }^{2}$. The oxygen content of each calcine was estimated using the method detailed by $\mathrm{O}^{\prime} \mathrm{Brien}^{3}$.

The mass of calcine in each layer $\left(\mathrm{M}_{\text {calcine }}\right)$ was calculated from the following equation:

$$
M_{\text {calcine }}=\rho_{\text {calcine }} V_{\text {calcine }}
$$

where $\rho$ and $\mathrm{V}$ are the assumed calcine density and tabulated volume for the layer. The composition of calcine in each layer was calculated from the component mass data provided in the calcine database as follows:

$$
M F_{i}=\frac{M_{i}}{M_{j}}
$$

where $\mathrm{MF}_{\mathrm{i}}$ and $\mathrm{M}_{\mathrm{i}}$ are, respectively, the mass fraction and total mass of the ith component for a given layer. No other changes were made to the existing calcine database.

\subsection{Calcination Process Mixing}

The data in the calcine database discussed in Section 2.3.1 provides useful information about the calcine on a batch-by-batch basis. However, the data does not account for mixing of calcines from different batches that occurred during calcination and deposition in the binsets and additional mixing which will occur when the calcine is extracted from the binsets. These mixing processes are described briefly below. 


\subsubsection{Mixing in the Calciner}

The volume of calcine in the calciner vessel is roughly $150 \mathrm{ft}^{3}$. Assuming the calciner behaves as a continuously stirred tank reactor (CSTR), once the feed to the calciner changes it requires several turnovers of the calciner inventory before the first feed is fully purged. In the interim the two feeds are blended such that the calcine composition continuously varies between pure feed 1 and pure feed 2 in decaying exponential fashion. Thus, the interface between the two calcines corresponding to the two different feeds is actually "smeared" (i.e., mixed) enroute to the binsets.

\subsubsection{Mixing Upon Deposition in the Bins}

After leaving the calciner, the calcine is pneumatically transported to one of the binsets. On reaching the top of a binset the calcine is extracted from the transport air in a cyclone and it falls by gravity through ducts into one (or more) of the bins within the binset. As it falls into the center of a bin it mounds into a conical pile. The calcine particles tumble down from the apex of the cone until coming to rest somewhere on the conical surface. The tumbling calcine particles impart momentum to the calcine that is already deposited, resulting in intermixing of stationary calcine with falling calcine. This effect also tends to "smear" (mix) the interfaces between strata in the bins containing different calcine types.

\subsubsection{Mixing During Retrieval}

As described in Section 3.1 the accepted method of retrieval of calcine is to fluidize a portion of the top layer of calcine with a high-velocity air stream and then suck this fluidized calcine into a vacuum nozzle. This scheme results in smearing of the interfaces between calcine layers as a result of two mechanisms. First, it is assumed that the suction nozzle would operate from a pivot in the center of the bin so as to trace out a hemispherical surface in the calcine. Since the calcine is deposited in conical layers, this means that the suction nozzle would effectively sweep through two (or more) calcine layers when it reaches an interface. Thus, the calcines near the interface are mixed. The second mixing mechanism results from the blowing action of the fluidizing nozzle. Since not all the calcine in suspension at a given time will be intercepted by the suction nozzle, some calcine particles fall back onto the top of the calcine column, stirring up the stationary particles, as during the deposition process, described above. Again, this results in some smearing of the interfaces between the calcine layers.

\subsubsection{Mixing Impacts}

As result of the above mixing mechanisms, sharp discontinuities would not be observed in the "average" composition of calcine reaching a mixing bin after retrieval. Rather, the composition would vary continuously between the compositions represented by the strata in the calcine database. The exact manner in which this variation would occur is a very complex function of the mechanisms described above and an accurate, mechanistic description of the composition variation in the retrieved calcine as it comes from the bins would be difficult to construct. However, the effect of the mixing

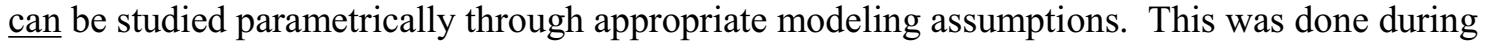
selected simulation studies described in the remainder of this report (see Section 4.2.3). 


\section{CALCINE RETRIEVAL AND BLENDING \\ 3.1 Retrieval}

\subsubsection{Retrieval Methods}

The current plan for retrieval will use pneumatic transport to move calcine from the bins/binsets to the treatment facility. This retrieval method has been simulated at full scale (for typical single bins and binset 1) and appears to work well when used to lift and transport free-flowing simulated calcine. The retrieval procedure involves the following steps:

- Build a remote pneumatic system atop each binset;

- Penetrate each bin in the binset in turn with a "stinger";

- Fluidize the top layer of calcine with blower nozzle and pull air/calcine suspension through a suction nozzle;

- Articulate the nozzles remotely using closed circuit cameras;

- Shield the pneumatic transfer lines and move retrieved calcine to a blending vessel;

- Homogenize calcine and characterize;

- Move blended calcine to treatment facility.

\subsubsection{Previous Retrieval Studies}

Three studies have examined methods to retrieve and transport calcine from the binsets. These studies are described briefly below.

\subsubsection{Raytheon Study}

Binset 1 was the first structure built at INTEC to store radioactive calcine. Its design is significantly different from those of the other binsets. It consists of a rectangular concrete vault which houses four stainless steel bins. Each of these four bins is a composite of three concentric bins, two annular and one cylindrical. The age and unique features of binset 1 have raised questions in recent years about the adequacy of its seismic design in the event of an earthquake. As a result, methods have been considered to remove the calcine stored in binset 1 and move it to a more modern facility. In 1995 Raytheon Engineers and Constructors, Inc. developed an access method to retrieve calcine from this binset. This work is documented in Ref. 4.

\subsubsection{Fluor-Daniels Study}

A second study of calcine retrieval systems was performed by Fluor-Daniels in 1997. This work built upon the Raytheon study and provided a design which provided the basis for a later study (see Section 3.1.2.3, below). This study is documented in Ref. 5.

\subsubsection{S.E. Gifford Study}

The most recent documented study of calcine retrieval systems was completed in 1998 by S.E. Gifford (Ref. 6) as part of a comprehensive effort to describe calcine treatment options to comply with the 1995 Settlement Agreement. The scope of this study was limited to include only those 
modifications to the Fluor-Daniels retrieval system design which were necessary to accommodate the unique needs of the five calcine treatment options under consideration at the time. The study detailed design requirements and all the required steps to construct, operate, and maintain a pneumatic calcine retrieval and transport system. The basic components for this system consisted of the following items:

- A new set of risers installed into the stainless steel bins through which access to the calcine could be obtained. These would be installed after drilling of penetrations through the roofs of the concrete shielding vaults of the binsets;

- A two-nozzle pneumatic system utilizing a pressurized air jet to fluidize calcine in the bins and a suction nozzle to retrieve the air-suspended calcine. This system was to be mated with the retrieval risers described above. This system incorporated a vertical deployment apparatus to position the nozzles at the surface of the calcine, and to allow attachment of additional sections to the nozzle lines as the level of the calcine in the bins dropped;

- A transport system to move calcine from the retrieval apparatus atop the binsets to the facility where it would be treated. This system would also be pneumatic and would couple to the retrieval system atop each binset. It would include "booster" stations (as needed) to convey the air-suspended calcine over the distance from the binsets to the treatment facility. The transport system would incorporate two independent subsystems, each capable of transporting $2,700 \mathrm{~kg} / \mathrm{hr}$ of calcine under vacuum. The vacuum requirement was to minimize the discharge of radioactive calcine in the event of a breached transport line.

Two of the treatment options under consideration at the time the study was completed required blending from all the binsets while the remaining options did not have this requirement. Thus, two different systems were examined - one with only two suction lines which could be moved from binset to binset, and one with a separate suction line for each binset, suggesting the possibility of drawing calcine from any binset at any given time. However, both these systems were designed to fully empty each bin within a binset before moving to a new bin. The study postulated approximately one week to move a calcine retrieval system from one binset to another, and approximately one day to move to a new bin within a binset.

The total unescalated cost estimate for the complete system with separate retrieval systems supplied for every binset varied from $\$ 178 \mathrm{M}$ to $\$ 243 \mathrm{M}$, depending on the length of the required transport system lines and the need for booster stations between the binsets and the treatment facility. Reference 6 indicates that preliminary cost estimates were done for both the 2-suction-line system and the system with simultaneous retrieval from all binsets, and that the capital cost estimates for these two systems did not differ significantly.

\subsection{Optimized Retrieval with Blending}

An ideal calcine retrieval and blending system would consist of a single blending bin large enough to hold all the calcine in all the binsets. All the calcine would be transferred, one bin at a time, into this large bin and then blended into a single homogeneous batch, characterized by a single average composition. This blended calcine would be fed to the calcine treatment process. However, the large volume of the calcine $\left(>4,000 \mathrm{~m}^{3}\right)$ makes design of such a large blending bin impractical or impossible. Therefore, the ideal system must be approached piecemeal, using a small blending bin, and selecting calcines from multiple bins to be blended into small, discrete batches. To approximate 
the performance of the ideal system calcine portions are chosen for each blended batch in a way that the following objectives are realized:

- The average composition of each blended batch is as close as possible to what the large "ideal" blender would produce if it were available;

- Subsequent blended batches can also be produced which also have average compositions approaching that of the ideal blender;

- If all subsequent batches cannot approach the composition of the ideal blender, then the number with "large" composition deviations is small.

To realize these objectives a number of variables can be adjusted, depending on the retrieval scenario assumed. These include the following:

- The amounts of calcine drawn from the available bins to produce a given blended batch;

- The number of bins used to supply calcine for a given blended batch (i.e., the number suction points available to provide calcine to the blending bin);

- The order of draining the bins within a binset;

- The order of draining of the binsets;

- The size of the blending bin. (However, as implied above, there is a physical limit to the batch size that can physically be blended prior to treatment. Concrete is typically mixed and transported in roughly $3-5 \mathrm{yd}^{3}$ batches so it seems reasonable that a stationary blending unit might accommodate up to $10 \mathrm{~m}^{3}$ of solids.)

By changing these variables an infinite variety is achievable in the range of blended calcine compositions which could emerge from the blending bin. This variety is of interest here, since the objective is to minimize the variation of composition in the calcine fed to the treatment process. Such minimization can significantly reduce the cost of treating the calcine, as it minimizes the number of flowsheets that the treatment process equipment must accommodate and reduces the number of waste forms that must be qualified for ultimate disposal. However, the capability to vary all retrieval parameters listed above requires a fairly complex and costly retrieval system. There is clearly a tradeoff between retrieval system hardware costs on one hand and development and qualification costs for process flowsheets and waste forms on the other. A more-complicated (and costly) retrieval system allows more flexibility in minimizing composition variations of blended, retrieved calcines, but the savings associated with simpler process flowsheets and fewer waste forms may not be sufficient to justify the higher retrieval system cost.

In the present study three retrieval system scenarios were considered. Each of these scenarios represents a different degree of complexity of retrieval system hardware. Each differs in the number of suction points that would be available for calcine and in the order in which the calcine is retrieved from within each binset. These scenarios were presumed to span most (but not all) of the spectrum of possibilities defined by the retrieval variables listed above. The three scenarios were as follows:

- Scenario 1 (43-bin blending): In this scenario all bins in the binsets are available to provide calcine for any batch of mixed calcine. The only constraint is that calcine must be drawn from the topmost layer in each bin at any given time (i.e., the suction point in a bin cannot be placed inside the column of calcine; rather, it must be on the prevailing top 
surface in the bin at the time). Of the three cases considered this scenario is the least limiting to the range of blended compositions achievable in the blending bin. Thus it provides the most latitude in accomplishing the desired objective.

- Scenario 2 (6-bin blending): For this scenario one bin per binset can provide calcine to any given batch. In addition, calcine from another bin in the same binset cannot be drawn until the bin currently being retrieved is empty. Thus, a maximum of 6 bins are available for forming the blended calcine batches until entire binsets begin to empty (then there are fewer). This case was moderately limiting to the range of blended compositions achievable in the blending bin, being between Scenarios 1 and 3.

- Scenario 3 (2-bin blending): In this scenario one bin in each of two binsets can supply calcine. Subsequent bins in the same binset can be tapped only when the current bin empties. In addition, other binsets cannot be tapped until one of the two current binsets is fully emptied. Thus, a maximum of 2 bins are available for forming the blended calcine batches at any given time. This case was most limiting to the range of blended compositions achievable in the blending bin.

It should be noted that Scenarios 2 and 3 correspond to the retrieval systems described (and costed) in reference 6 . 


\section{RETRIEVAL OPTIMIZATION STUDIES}

\subsection{Background}

\subsubsection{Introduction}

Optimization of the retrieval process to achieve the stated objective (minimization of the variation in composition of the blended calcine) by varying the retrieval variables listed in Section 3.2 represents a formidable challenge. This, together with the fact that the current study was intended to be scoping in nature, dictated that simplifying assumptions be made. The first of these assumptions was to assume that each blended batch of calcine would be made by mixing a fixed (but arbitrary) number, N, of discrete portions of calcine. All such portions would contain a fixed mass. (Except when the quantity of calcine remaining in a bin was less than the specified mass; in this case the portion size for that bin was equal to the remaining calcine in the bin.) Each equal-size calcine portion would be drawn entirely from a single bin. For clarity in the remainder of this report, each discrete portion is referred to as a "chunk", to emphasize the fact that it came from a single location in a single bin. In summary, the first simplifying assumption was to restrict the retrieval and blending operation to choosing $\mathrm{N}$ equal-sized portions ("chunks") of calcine from the available bins to make a blended batch in the blending bin. The available bins for retrieval would depend on which of the three blending scenarios discussed in Section 3.2 was being considered.

The second simplying assumption was to fix the size of the blending bin to $10 \mathrm{~m}^{3}$ for all retrieval simulations. With this batch size, it takes 441 batches to empty and process the calcine in all the binsets.

The third simplifying assumption was to fix ' $N$ ' (the number of "chunks" used to make a blended batch of retrieved calcine) at 10 .

Given these three simplifying assumptions, the three retrieval scenarios described in Section 3.2 still allow considerable latitude in mixing chunks of calcine from different bins to form $10-\mathrm{m}^{3}$ batches. All of the scenarios are computationally difficult because of the large number of possible mix compositions. For example, in Scenario 1, retrieving 10 portions from up to 43 bins to form a single batch can be done nearly $43^{10}$ (i.e., $2 \times 10^{16}$ ) ways. Moreover, this process must be repeated for each of the over-400 batches needed to empty all the binsets. Clearly, an investigation of all of these possible batch recipes is beyond the limits of time and computing resources. Hence, the number of recipes must be reduced to allow computation. This reduction can be accomplished by either intelligently paring the combination tree to investigate only promising batch recipes, or by randomly selecting batch recipes and comparing them to find the best of those tested. Because of limited time and the availability of cheap computing resources (i.e., fast desktop PCs) we chose not to attempt to define a paring scheme, but rather to pursue the random approach, commonly termed a "Monte Carlo" simulation. This approach, while not as elegant as the former, presented an opportunity to generate, in a fairly short time, a reasonable engineering solution to the problem.

\subsubsection{Basic Monte Carlo Model}

In this approach the basic idea is to form (computationally, with a retrieval simulation program) a large number of randomly-selected trial batches of mixed calcine from the available bins and compare the batches to find the one best meeting the desired objective. For the problem at hand this meant selecting the batch which minimized an optimization function, $\phi$. The optimization function was selected to represent with a single number 'the variation of the blended batch composition from the mean over the entire calcine inventory'. The definition of such a function is 
somewhat arbitrary, and two such functions were tried in the course of the study. (The bases for defining these functions is described later.)

The retrieval simulation program generates a retrieval recipe which is approximately optimal in the sense of minimizing the optimization function. This recipe defines a retrieval sequence through all the bins consisting of 441 batch prescriptions. A single batch prescription is a set of $\mathrm{N}$ bin identifiers indicating the bins used to provide the $\mathrm{N}$ chunks for that batch. Each batch prescription is selected using the Monte Carlo process described in the preceding paragraph. Thus, 441 of these Monte Carlo selections are performed to define a single candidate retrieval recipe. The process is repeated to define many candidate retrieval recipes, which are then compared to choose the one which is optimal in the sense that the retrieval recipe objective function, $\psi$, is minimal. (Note the distinction between $\psi$, and the batch optimization function, $\phi$, defined above). The retrieval recipe objective function, $\psi_{\mathrm{j}}$, for the jth candidate retrieval recipe is defined as follows:

$$
\psi_{j}=\max \left\{\phi_{i} \mid \phi_{\mathrm{i}} \in(\text { single batch objective functions for jth retrieval recipe })\right\}
$$

Because of the many retrieval recipes possible, only a small fraction can be calculated, given the time and computing resources available. Thus the "best" retrieval recipe found by the above procedure is unlikely to be the best when all possible retrieval sequences are considered, and the success of the method relies on the assumption that there are many "good" plans, some of which will be uncovered by the random search. The simulation is completely random; that is, no knowledge of binset or bin contents is used to influence the choice of trial retrieval schemes. The choice of retrieval recipes is constrained only by the assumptions of the retrieval scenario being modeled.

The Monte Carlo retrieval simulation program was written in the " $\mathrm{C}$ " language. A brief overview of the calculation scheme used by the program is given below as descriptive pseudocode.

\section{SIMPLIFIED ENGLISH PSEUDOCODE}

User inputs - Number of trials used to generate each batch prescription;

Number of candidate recipes used to generate the optimum recipe;

Name and path of data file containing bin calcine composition data;

Random number seed for Monte Carlo calculations

Read calcine composition data file

FOR (number of recipes) DO:

Initialize recipe data

FOR (number of batches per recipe) DO:

Initialize batch data

FOR (number of random trials per batch) DO:

Select a random batch of chunks from random bins

Apply goodness-of-fit criteria to average batch composition

IF (This is the best batch recipe so far) THEN:

ENDIF

Record Best Batch information

END random trials loop

Record best batch recipe info into final recipe array

IF (any bins emptied forming this batch) THEN:

ENDIF

Remove empty bins from active bin list 


\section{END batches loop}

Apply best-fit criteria to entire recipe

IF (This is the best recipe found so far) THEN:

Record new best recipe data(write recipe and composition output files)

\section{ENDIF \\ END recipes loop \\ STOP}

The outermost loop in the pseudocode, with each pass generating a unique recipe for calcine retrieval, was typically repeated upwards of 20,000 times, occasionally over 200,000 times. After each recipe was generated it was compared to the best found to date, using the objective function, $\psi$, described above. When the program was modified to count the number of times that a new "best" recipe was found, it was noted that after the first 7 or 8 best recipes, new best recipes were found with decreasing frequency. For example, if the $8^{\text {th }}$ best recipe was found after 20,000 recipe iterations, the $9^{\text {th }}$ might not be found until 40,000 to 60,000 recipes were generated and compared.

Later versions of the program were made more efficient by applying the "best recipe" test to each of the 441 batches as they were generated. When a batch was found that failed the comparison with the best recipe (to date), the recipe calculation was terminated and calculation of the next recipe begun.

The "C" program generates two files for the best recipe found: 1) a recipe file that gives the bin number from which each chunk of calcine in each mixed batch was drawn, and 2) a list of the average mass fractions of aluminum, zirconium, and fluoride in each mixed batch. As an independent verification of the calculation, the recipe file was used as input to a separate Visual Basic (VB) program (the "C" and VB programs were authored by different people) that recalculates the composition of each of the 441 mixed batches of calcine in the retrieval recipe. Comparison of the calculated concentrations of aluminum (Al), zirconium $(\mathrm{Zr})$, and fluoride $(\mathrm{F})$ from the two programs identified inconsistencies which were subsequently resolved by analyzing and debugging the Monte Carlo "C" program. The choice of $\mathrm{Al}, \mathrm{Zr}$, and $\mathrm{F}$ for validation was dictated by their use in the optimization functions (as described later). In addition to providing a check on the simulation results, the VB program calculates batch concentrations of all other species identified as components of the calcine, and so is used to evaluate composition variations of components that were not included in the optimization scheme.

\subsubsection{Modifications to Basic Monte Carlo Model}

Applying the basic Monte Carlo approach to the second and third retrieval scenarios listed in Section 3.2 required some slight modifications. First, it was necessary to constrain the number of bins available to supply calcine to each batch to a number less than the total number of bins. Second, it was necessary to install coding that would switch to a new bin in each binset when the current bin was emptied. Third, it was necessary to track temporary bin changes that would naturally occur during the random searches (e.g., when a bin emptied) and reset all bins to the original configuration at the start of the batch for each random trial. (Similar tracking was required for entire binsets to maintain accurate accounting of which binsets were still available to supply calcine at any given time, an important consideration as calcine was exhausted and the simulation approached the $441^{\text {st }}$ batch.)

On the basis of early calculations which were done, a fourth change was made to improve the efficiency of the random search, and thus speed up the simulation. As a result of this change a recipe would not be completed if the concentration variation for any species in a "best" batch exceeded the worst variation in the best recipe found to date. Thus it was possible that only a few of the 441 
batches per recipe would be calculated for recipes inferior to the best already found. A significant increase in calculation speed was noted as a result of this change.

In addition to the above modification a change in user input was also found to accelerate the calculations. This was discovered through an informal sensitivity study which suggested that calculation efficiency increased when the number of random trials per batch was reduced, allowing a greater number of complete 441-batch recipes to be generated in the same amount of time. As a result, 40 random trials per batch were used instead of the 1000-trials-per-batch used in previous studies. These two changes made it possible to calculate over one million trial recipes per day of calculation, rather than 20,000 to 30,000 generated previously during two or three-day runs. Specifically, a $550 \mathrm{MHz}$ Pentium computer completed one million recipe trials in 13 hours. Interestingly, the best recipe was found after only about 90,000 trials - the following $900,000+$ trials could find no better.

In addition to the above, some additional modifications to the random search algorithm were necessitated by the second and third retrieval scenarios. These changes were considered minor and are not described here. Other changes involved coding different choices for the optimization function. The optimization functions used for the three retrieval scenarios are described in Sections 4.2.1.1 and 4.2.3.1, below.

\subsection{Optimization Studies}

\subsubsection{Phase 1 Study}

The Phase 1 study was performed first. It was directed at answering the question, "Does retrieval optimization offer a sufficient benefit to warrant investigating it further?" That is, in the absence of cost, safety, or operational constraints on the retrieval system, could optimized retrieval and blending provide a significant benefit in the treatment of the calcine and the qualification of the final waste forms. Thus, for the Phase 1 optimization study the first of the three retrieval scenarios in Section 3.2 (i.e., the least restrictive one) was selected as a starting point. If the answer to the above query had turned out to be "No" additional studies would not have been pursued.

\subsubsection{Assumptions}

Specific assumptions applied in setting up the Phase 1 study are as follows:

- Discrete chunks of calcine could be retrieved from any of the 43 bins in the six binsets. Thus, a calcine retrieval line is available in every bin of each binset at all times.

- Different calcines described in the database discussed in Section 2.3.1 reside in distinct horizontal layers, unmixed with the calcines above and below. Thus, as calcine is retrieved, starting at the top of each bin and proceeding to the bottom, abrupt changes in calcine composition occur at the interfaces between successive layers.

- The blending bin for retrieved calcine will accommodate $10 \mathrm{~m}^{3}$.

- The volume of each calcine chunk is $1 \mathrm{~m}^{3}$. Thus, a recipe for a single batch of mixed calcine would specify 10 bins to provide the $1-\mathrm{m}^{3}$ chunks to the mixing vessel. (Note, however, that any given bin could supply more than one of the 10 chunks for a batch.) 
- The total amount of calcine in the binsets was taken from the Excel spreadsheet described in Ref. 1 as constituted on 1/1/2000. Based on this information, 441 mixing batches $\left(10 \mathrm{~m}^{3}\right.$ each) were required to empty the binsets.

- The retrieval system will withdraw all of the calcine from every bin - none remains after a bin is emptied.

- The optimization function for which a minimum was sought is the sum of the deviations of key species from their average values, mathematically expressed as follows:

$$
\phi={ }_{i}\left[\frac{\left(M F_{i}-\overline{M F}_{i}\right)}{\overline{M F}_{i}}\right]^{2}
$$

where "MF" indicates mass fraction of specie "i", overbar indicates the average over all calcines in all binsets, and the summation index "i" includes only $\mathrm{Al}$ (aluminum), $\mathrm{F}$ (fluoride), and $\mathrm{Zr}$ (Zirconium).

In defining the above optimization function, $\mathrm{Al}, \mathrm{F}$, and $\mathrm{Zr}$, were selected as the species whose variations are most likely to impact the number of required glass recipes required to vitrify calcine. These species were selected after conversations with glass specialists at INEEL (Chris Musick), the Savannah River Site (David Peeler), and Pacific Northwest National Laboratory (John Vienna).

\subsubsection{Results}

The results of the simulations for the Phase 1 study are shown in Figure 4-1 and Figure 4-2. They show batch-by-batch composition variations for the "best" retrieval recipe found by the Monte Carlo simulation (the $\mathrm{x}$-coordinate is the sequential batch number as the bins are emptied; the $\mathrm{y}$ coordinate shows the mass fractions of the respective elemental components of the calcine, as discussed in Section 2). The data for the figures was generated using the VB program described in Section 4.1.2. The major species, $\mathrm{Al}, \mathrm{Zr}$, and $\mathrm{F}$, used to define the optimization function, and $\mathrm{Ca}$ are shown in the first two plots of Figure 4-1. Selected minor elemental constituents of the calcine are shown in the third plot of Figure 4-1 and in the three plots of Figure 4-2.

Statistical measures of the batch-by-batch composition variation are shown below in Table 4-1 and Table 4-2. Entries include:

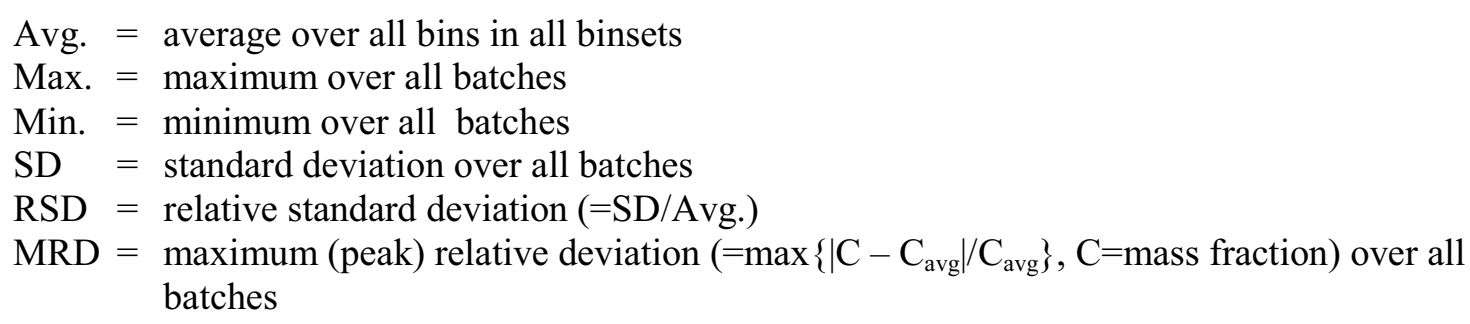
batches

Table 4-1 gives the statistics for the three components used in the optimizing function (Al, $\mathrm{Zr}$, and $\mathrm{F}$ ) and Table 4-2 gives statistical information for other components. To facilitate comparison of optimized and non-optimized retrieval results, composition variation statistics for $\mathrm{Al}, \mathrm{Zr}$, and $\mathrm{F}$ from the non-optimized retrieval scenario described in Section 1.2 are given in Table 4-3. 
The relative standard deviation (RSD) of the mass fractions for $\mathrm{Al}, \mathrm{Zr}$, and $\mathrm{F}$ were $2 \%, 3 \%$ and $3 \%$, respectively. Corresponding RSD values for the non-optimized retrieval scenario discussed in Section 1.2 were $46 \%, 38 \%$, and $35 \%$. Thus, optimization of the retrieval process has reduced the RSD values by factor of 19,14 , and 12 , respectively. The peak relative deviations (MRDs) from the mean composition were $13 \%, 14 \%$, and $17 \%$ for $\mathrm{Al}, \mathrm{Zr}$, and $\mathrm{F}$, respectively, while corresponding values from the non-optimized retrieval scenario of $138 \%, 81 \%$, and $84 \%$, respectively. These results indicate that optimized retrieval of the calcine can significantly reduce the composition variation of blended calcine fed to the treatment process.

Having thus answered the question posed in Section 4.2.1 affirmatively, following completion of the Phase 1 study we proceeded to optimization studies of less complex (less costly) retrieval systems. These were the Phase 2 and 3 studies, discussed below.

Table 4-1: Statistics of composition variation of optimized species (Phase 1 study).

\begin{tabular}{|c|c|c|c|}
\hline Specie & Al & Zr & F \\
\hline Avg. & 0.1713 & 0.1025 & 0.1524 \\
\hline Max. & 0.1884 & 0.1119 & 0.1783 \\
\hline Min. & 0.1488 & 0.0878 & 0.1425 \\
\hline SD & 0.0041 & 0.0028 & 0.0046 \\
\hline RSD & $2 \%$ & $3 \%$ & $3 \%$ \\
\hline MRD & $13 \%$ & $14 \%$ & $17 \%$ \\
\hline
\end{tabular}

Table 4-2: Statistics of composition variation of other species (Phase 1 study).

\begin{tabular}{|l|c|c|c|c|c|c|c|c|}
\hline Specie & Ca & B & Cd & Cr & Fe & Hg & K & Mg \\
\hline Avg. & 0.2044 & 0.0073 & 0.0077 & 0.0016 & 0.0039 & 0.0008 & 0.0046 & 0.0051 \\
\hline Max. & 0.2311 & 0.0098 & 0.0239 & 0.0020 & 0.0110 & 0.0048 & 0.0088 & 0.0165 \\
\hline Min. & 0.1665 & 0.0063 & 0.0000 & 0.0010 & 0.0017 & 0.0000 & 0.0010 & 0.0002 \\
\hline SD & 0.0099 & 0.0005 & 0.0056 & 0.0002 & 0.0013 & 0.0010 & 0.0015 & 0.0028 \\
\hline RSD & $5 \%$ & $7 \%$ & $73 \%$ & $10 \%$ & $32 \%$ & $131 \%$ & $33 \%$ & $56 \%$ \\
\hline MRD & $19 \%$ & $35 \%$ & $212 \%$ & $35 \%$ & $182 \%$ & $519 \%$ & $91 \%$ & $226 \%$ \\
\hline
\end{tabular}

\begin{tabular}{|l|c|c|c|c|c|c|c|c|}
\hline Specie & Mn & Na & Nb & Ni & Sr & Sn & PO4 & SO4 \\
\hline Avg. & 0.0002 & 0.0212 & 0.0005 & 0.0005 & 0.0021 & 0.0016 & 0.0044 & 0.0091 \\
\hline Max. & 0.0010 & 0.0370 & 0.0037 & 0.0007 & 0.0025 & 0.0018 & 0.0378 & 0.0201 \\
\hline Min. & 0.0000 & 0.0050 & 0.0000 & 0.0002 & 0.0016 & 0.0014 & 0.0007 & 0.0021 \\
\hline SD & 0.0002 & 0.0053 & 0.0009 & 0.0001 & 0.0001 & 0.0000 & 0.0054 & 0.0037 \\
\hline RSD & $100 \%$ & $25 \%$ & $168 \%$ & $17 \%$ & $6 \%$ & $3 \%$ & $125 \%$ & $40 \%$ \\
\hline MRD & $346 \%$ & $76 \%$ & $578 \%$ & $50 \%$ & $23 \%$ & $13 \%$ & $768 \%$ & $120 \%$ \\
\hline
\end{tabular}

Table 4-3: Statistics of composition variation of Al, Zr, and F for non-optimized retrieval.

\begin{tabular}{|l|c|c|c|}
\hline Specie & Al & $\mathbf{Z r}$ & F \\
\hline Avg. & 0.1656 & 0.1088 & 0.1609 \\
\hline
\end{tabular}




\begin{tabular}{|l|c|c|c|}
\hline Max. & 0.3947 & 0.1853 & 0.2471 \\
\hline Min. & 0.0619 & 0.0208 & 0.0254 \\
\hline SD & 0.0759 & 0.0416 & 0.0555 \\
\hline RSD & $46 \%$ & $38 \%$ & $34 \%$ \\
\hline MRD & $138 \%$ & $81 \%$ & $84 \%$ \\
\hline
\end{tabular}



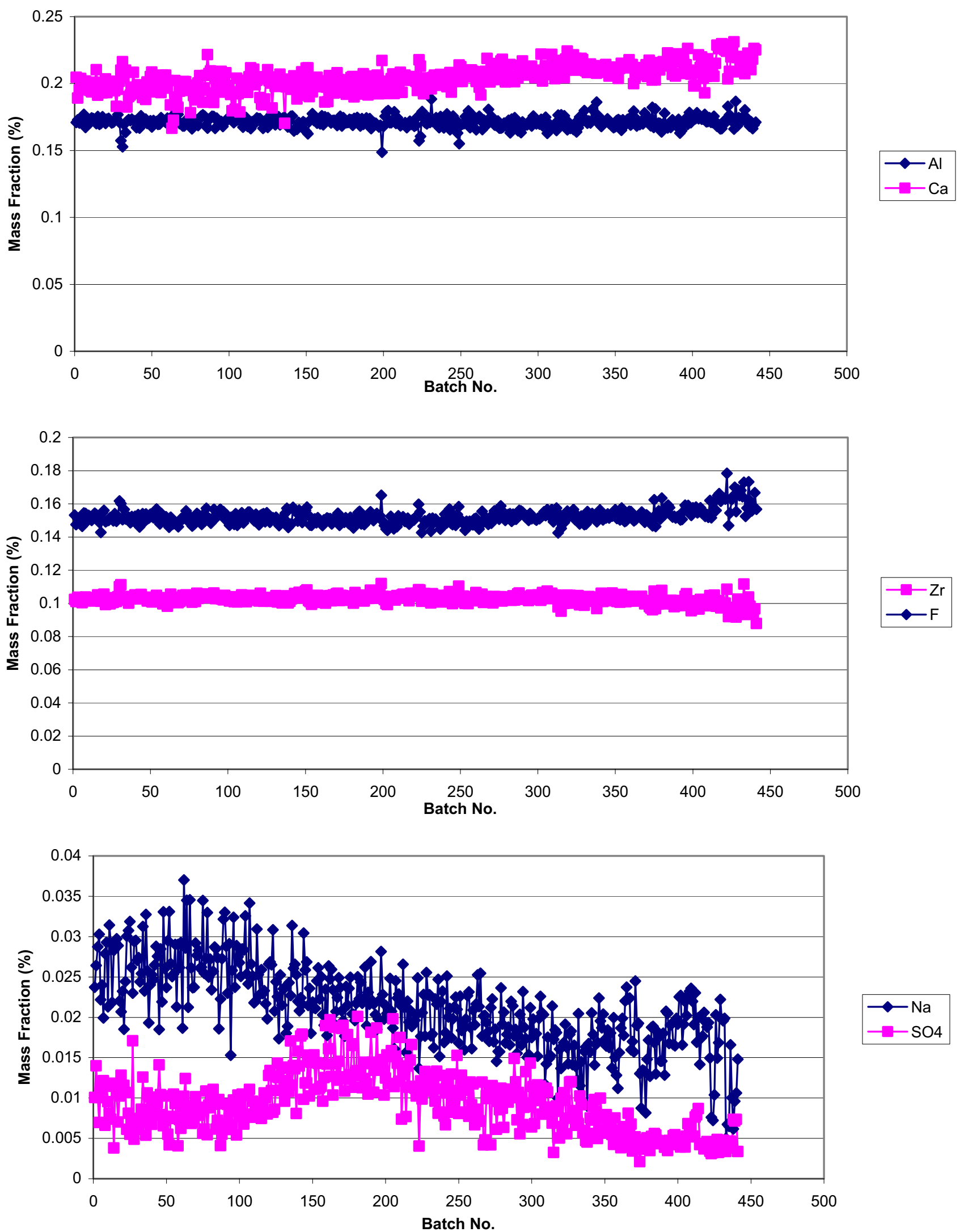

Figure 4-1: Batch-by-batch composition variation (major species, Phase 1 study). 

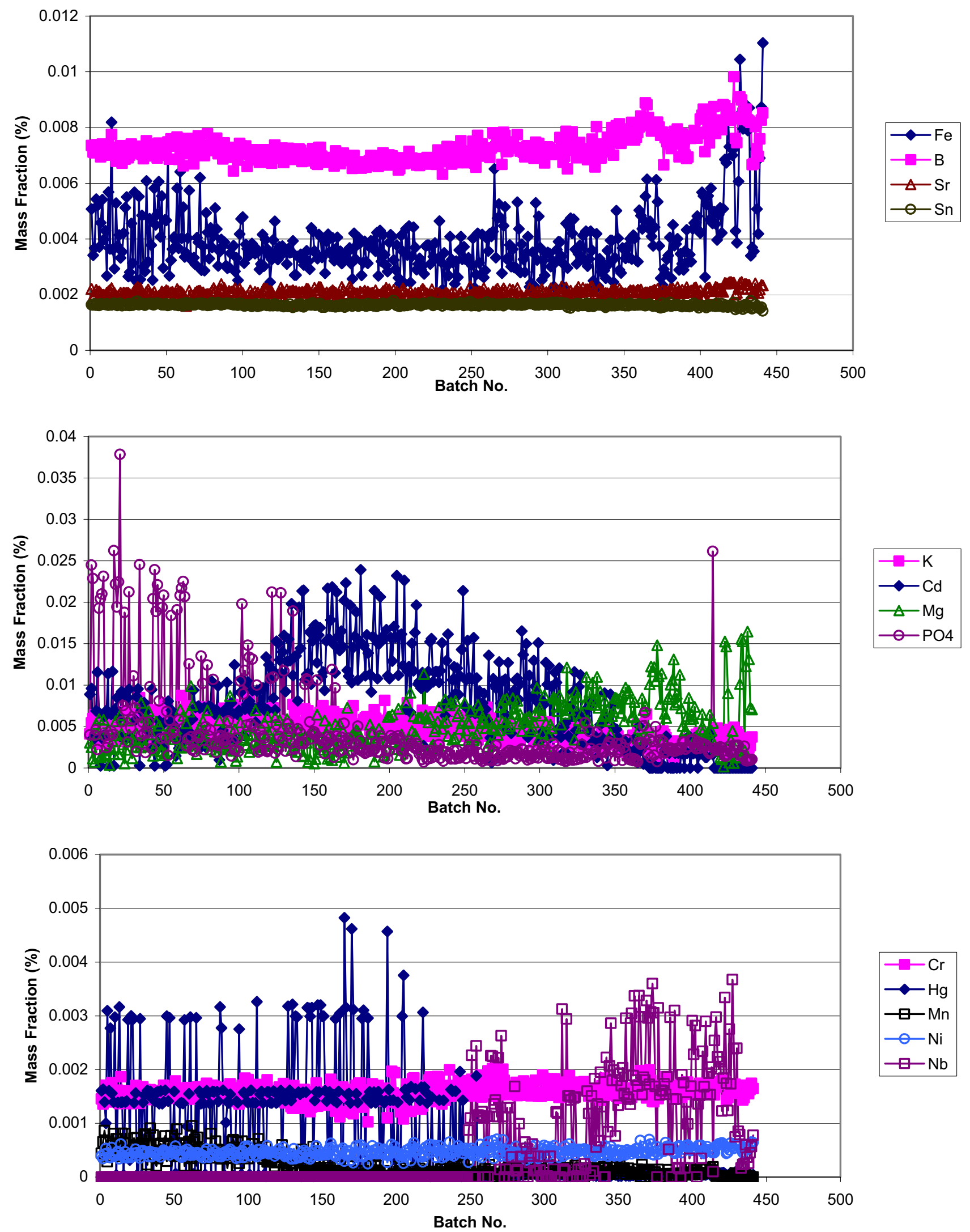

Figure 4-2: Batch-by-batch composition variation (major species, Phase 1 study). 


\subsubsection{Phase 2 Study}

The results of the Phase 1 study were presented and discussed with vitrification personnel from the INEEL, the Savannah River Site, and Pacific Northwest National Laboratory (PNNL) at a vitrification workshop held at the INEEL on February 23-24, 2000. These discussions led to a recommendation that the Phase 1 study assumptions be modified to reflect a more modest retrieval system than that suggested by the first retrieval scenario in Section 3.2. The Phase 2 study was thus directed at answering the same question as for the Phase 1 study, but with a retrieval system as described by the second retrieval scenario in Section 3.2.

\subsubsection{Assumptions}

Assumptions for the Phase 2 study are as follows:

- A single calcine retrieval line is available in each binset at all times.

- Once a binset's retrieval line has been inserted into a bin, it cannot be withdrawn until the bin is completely emptied. Thus, in general only one bin within a binset can supply calcine for a blended batch. (The only exception is that two bins within a binset can supply calcine to a batch when one bin is emptied during the course of creating the batch.)

- Calcine can be retrieved from the bins within a binset in any order.

- After an entire binset has been drained, its calcine retrieval line cannot be used on another binset. Thus, the "single retrieval line per binset" constraint is maintained through the entire retrieval process.

- The optimization function for which a minimum was sought was identical to that used in the Phase 1 study (see Section 4.2.1.1).

\subsubsection{Results}

As for the Phase 1 study, the output of the Phase 2 Monte Carlo simulation was a "best" retrieval recipe. This recipe was post-processed using the VB program discussed in Section 4.1.2. The results are shown below in Figure 4-3 and Figure 4-4. Statistical measures of the calculated batch-by-batch composition variation are shown below in Table 4-4 and Table 4-5. Note that the RSDs in Table 4-4 are smaller than those for the Phase 1 study in Table 4-1. This result is counterintuitive since the Phase 1 optimization was less constrained than that for the Phase 2 study. This is discussed further in Section 4.3.

The MRDs for this study were $5 \%, 7 \%$, and $10 \%$ for $\mathrm{Al}, \mathrm{Zr}$, and $\mathrm{F}$, respectively. These values are also lower than the corresponding values for the Phase 1 study and much below corresponding values for non-optimized retrieval.

Table 4-4: Statistics of composition variation of optimized species (Phase 2 study).

\begin{tabular}{|l|c|c|c|}
\hline Specie & Al & Zr & F \\
\hline Avg. & 0.1713 & 0.1026 & 0.1524 \\
\hline Max. & 0.1800 & 0.1063 & 0.1673 \\
\hline Min. & 0.1660 & 0.0953 & 0.1458 \\
\hline SD & 0.0018 & 0.0018 & 0.0034 \\
\hline RSD & $1 \%$ & $2 \%$ & $2 \%$ \\
\hline
\end{tabular}


Table 4-5: Statistics of composition variation of other species (Phase 2 study).

\begin{tabular}{|l|c|c|c|c|c|c|c|c|}
\hline Specie & Ca & B & Cd & Cr & Fe & Hg & K & Mg \\
\hline Avg. & 0.2044 & 0.0073 & 0.0077 & 0.0016 & 0.0039 & 0.0008 & 0.0046 & 0.0050 \\
\hline Max. & 0.2270 & 0.0095 & 0.0261 & 0.0021 & 0.0101 & 0.0042 & 0.0093 & 0.0221 \\
\hline Min. & 0.1651 & 0.0065 & 0.0000 & 0.0010 & 0.0012 & 0.0000 & 0.0000 & 0.0001 \\
\hline SD & 0.0092 & 0.0005 & 0.0060 & 0.0002 & 0.0014 & 0.0011 & 0.0015 & 0.0032 \\
\hline RSD & $4 \%$ & $7 \%$ & $79 \%$ & $11 \%$ & $36 \%$ & $134 \%$ & $33 \%$ & $64 \%$ \\
\hline MRD & $19 \%$ & $30 \%$ & $239 \%$ & $40 \%$ & $158 \%$ & $443 \%$ & $103 \%$ & $337 \%$ \\
\hline
\end{tabular}

\begin{tabular}{|l|c|c|c|c|c|c|c|c|}
\hline Specie & Mn & Na & Nb & Ni & Sr & Sn & PO4 & SO4 \\
\hline Avg. & 0.0002 & 0.0212 & 0.0005 & 0.0005 & 0.0021 & 0.0016 & 0.0044 & 0.0091 \\
\hline Max. & 0.0010 & 0.0355 & 0.0046 & 0.0007 & 0.0024 & 0.0017 & 0.0390 & 0.0215 \\
\hline Min. & 0.0000 & 0.0000 & 0.0000 & 0.0002 & 0.0016 & 0.0015 & 0.0004 & 0.0007 \\
\hline SD & 0.0002 & 0.0052 & 0.0009 & 0.0001 & 0.0001 & 0.0000 & 0.0053 & 0.0039 \\
\hline RSD & $93 \%$ & $25 \%$ & $172 \%$ & $19 \%$ & $7 \%$ & $2 \%$ & $122 \%$ & $43 \%$ \\
\hline MRD & $352 \%$ & $100 \%$ & $743 \%$ & $54 \%$ & $23 \%$ & $7 \%$ & $793 \%$ & $135 \%$ \\
\hline
\end{tabular}



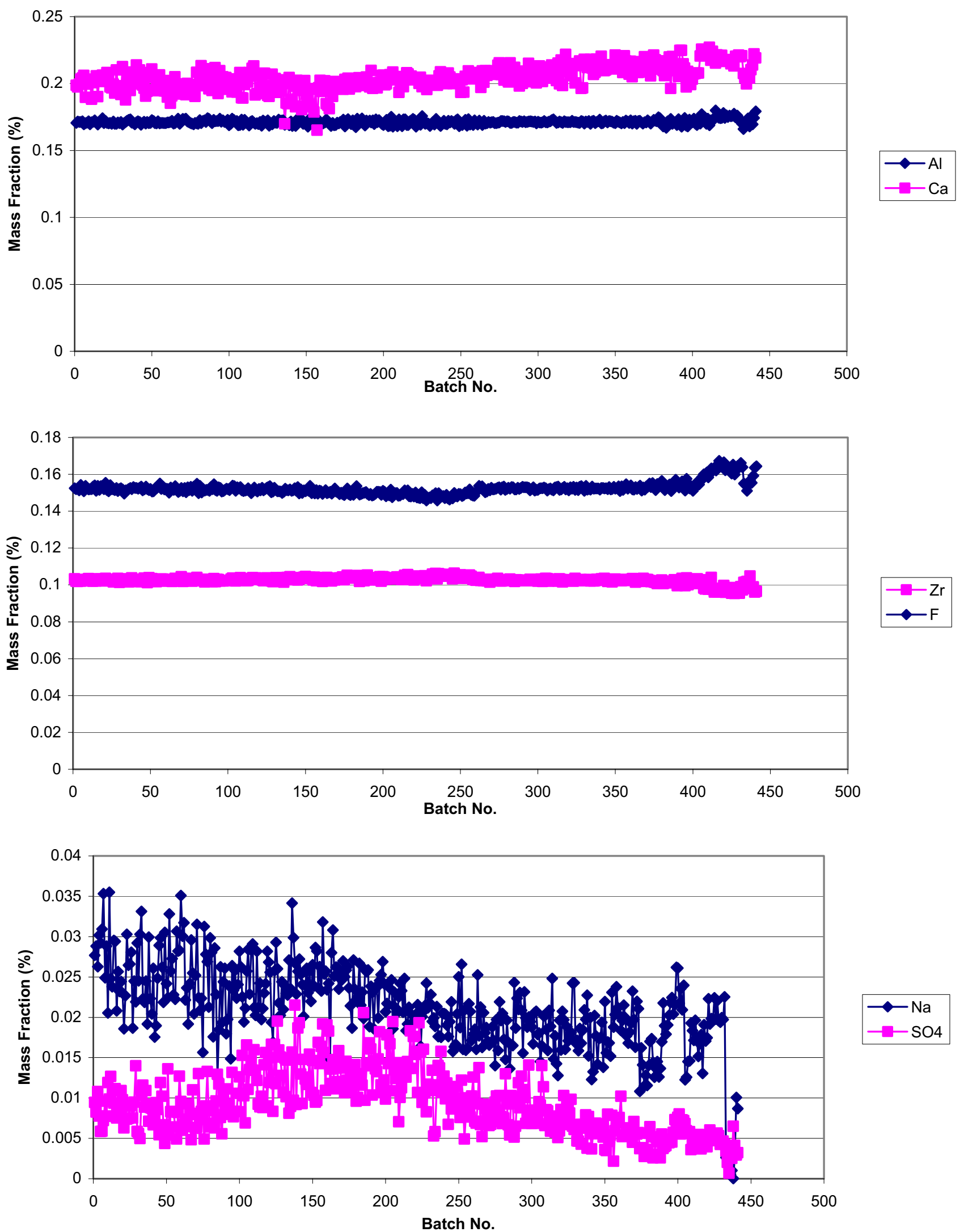

Figure 4-3: Batch-by-batch composition variation (major species, Phase 1 study). 

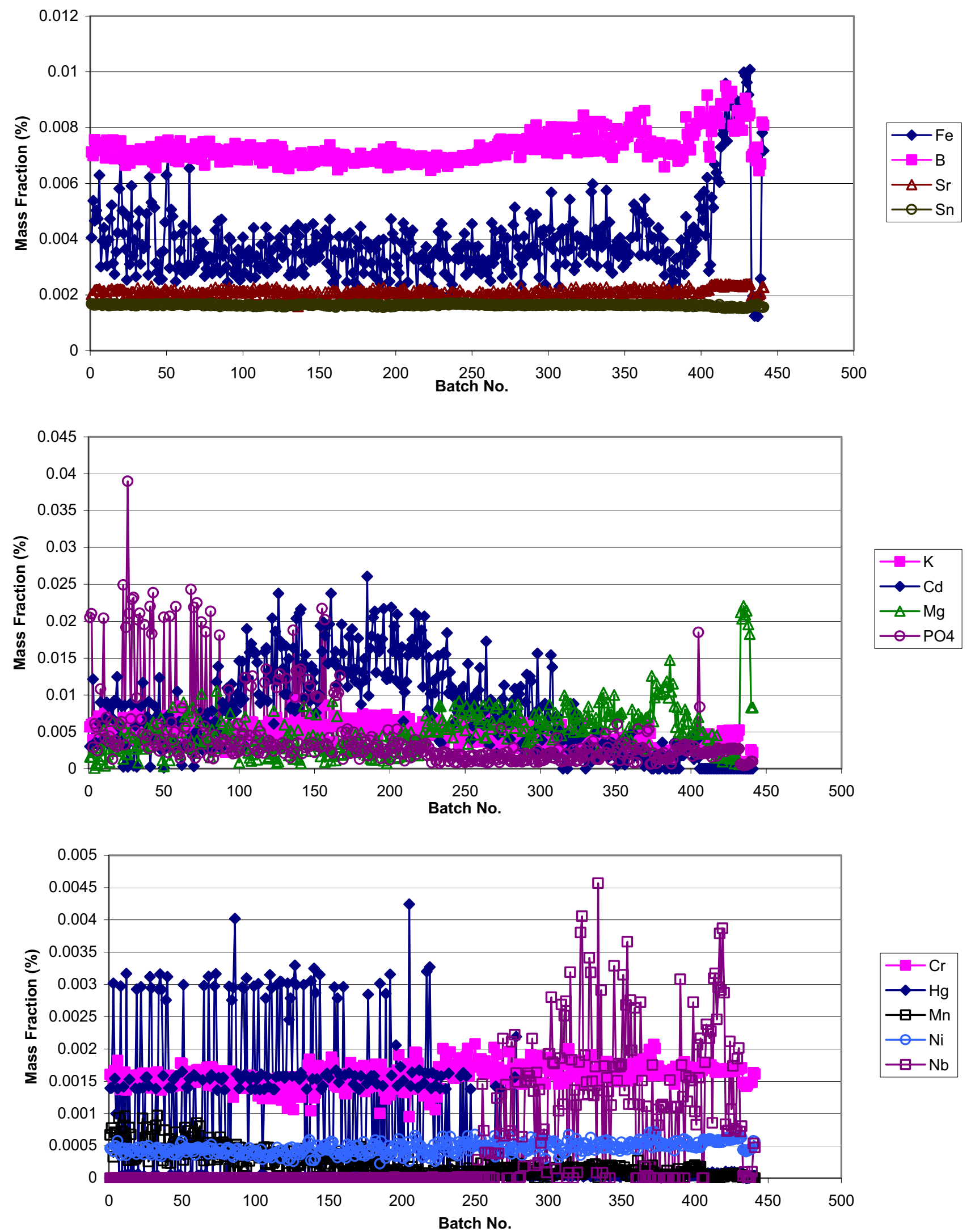

Figure 4-4: Batch-by-batch composition variation (major species, Phase 1 study). 


\subsubsection{Phase 3 Study}

The Phase 3 study involved further simplification (and reduced cost) of the retrieval system beyond what was assumed for the Phase 2 study. Specifically, the Phase 3 study was directed at answering the same question as for the Phase 1 and 2 studies, but with a retrieval system as described by the third retrieval scenario in Section 3.2. In addition, it was assumed that once a retrieval system was placed on a binset it had to remain with that binset until it was fully exhausted of calcine.

The vitrification workshop discussed in Section 4.2.2 also touched on the Phase 1 study assumption of perfect stratification in the bins. It was noted that the different calcine types would be intermixed before they reach the blending bin, by the mechanisms discussed in Section 2.4, and that this mixing could impact the conclusions of the optimization study. Therefore, we decided to perform a rudimentary sensitivity study of the effects of calcine mixing on blended calcine composition variation following retrieval. For this sensitivity study mixing of different calcines was represented mathematically by postulating a mixing zone of fixed (but arbitrary) thickness between each stratum in the bins. The mixing zone thickness was an input parameter in the simulation. Calcine within the mixing zone was mathematically redistributed to approximate a normal distribution about the geometric center of the mixing zone. The entire volume of calcine in each bin was discretized into thin layers so as to capture the continuous variation of composition between the strata. The thickness of these artificial layers was chosen so that the entire variation between successive physical strata was contained in nine (9) layers. These discrete layers were then used in calculating average compositions for each calcine chunk retrieved and batched to the blending bin.

Phase 3 simulations were performed both with and without the above mixing model.

\subsubsection{Assumptions}

Specific assumptions for the Phase 3 study are as follows:

- Exactly two suction lines are available to retrieve calcine.

- As in the Phase 2 study, once a bin is connected to one of the suction lines for retrieval it must be fully emptied before another bin can be connected for retrieval.

- Once a suction line is placed on a binset it must remain on that binset until all bins within the binset are fully emptied.

- After a binset is emptied, its suction line is moved to another binset, provided an unemptied binset is still available.

- At the suggestion of the vitrification workshop participants, the optimization function used in the Phase 3 study was changed from that used in the Phase 1 and Phase 2 studies. All compositions within a specified "band" about the mean were treated as being equally good. Compositions outside the band were treated in the usual sum-squares-deviation manner for mass fractions of Al, Zr, and F. Mathematically, the function which was minimized is expressed as follows:

$$
\begin{aligned}
& \phi=A_{i}^{2} \\
& A_{i}=\left\{\begin{array}{l}
0, \text { if }\left|M F_{i}-\overline{M F}_{i}\right|<B \\
\left|M F_{i}-\overline{M F}_{i}\right|-B, \text { if }\left|M F_{i}-\overline{M F}_{i}\right| \geq B
\end{array}\right.
\end{aligned}
$$


$\mathrm{B}$ is the half-band width of the acceptable concentration range about mean calcine species composition. Definition of B was implemented as a user input in the program, but for all calculations made using this function, B was set to 0.015 .

- In calculations where the pre-blending bin mixing model was used the model's mixing length parameter was assigned a value of $5 \mathrm{ft}$.

\subsubsection{Results}

Three calculations were made during the Phase 3 study. The first two (Cases A and B) were performed using mixed and unmixed calcine distributions, respectively, as was done previously for different retrieval schemes. To gain insight into the sensitivity of the results to calcine mixing, the third calculation used the best recipe found for unmixed calcine and applied it to the mixed calcine strata. The third calculation did not require use of the Monte Carlo program. It utilized only the Visual Basic simulation (where the mixing model described in Section 4.2.3.1 was applied). The optimal recipe obtained from Case A was used to direct the retrieval in the VB model. The calcine layers to which the recipe were applied, however, were mixed, using to the mixing model, prior to generating the blended batches of calcine.

The results of the three cases are summarized in the tables and figures which follow. Identical statistical tables and figures to those given for the Phase 1 and Phase 2 studies are provided only for Case A. For cases B and C, only the statistics and figures for variation of major species are presented.

\section{Case A (optimized retrieval with unmixed calcine layers as input to Monte Carlo model):}

Table 4-6: Statistics of composition variation of optimized species (Phase 3 study-Case A).

\begin{tabular}{|l|c|c|c|}
\hline Specie & Al & Zr & F \\
\hline Avg. & 0.1722 & 0.1026 & 0.1523 \\
\hline Max. & 0.2548 & 0.1631 & 0.2390 \\
\hline Min. & 0.0839 & 0.0522 & 0.0855 \\
\hline SD & 0.0290 & 0.0155 & 0.0187 \\
\hline RSD & $17 \%$ & $15 \%$ & $12 \%$ \\
\hline MRD & $51 \%$ & $59 \%$ & $57 \%$ \\
\hline
\end{tabular}

Table 4-7: Statistics of composition variation of other species (Phase 3 study-Case A).

\begin{tabular}{|l|c|c|c|c|c|c|c|c|}
\hline Specie & Ca & B & Cd & Cr & Fe & Hg & K & Mg \\
\hline Avg. & 0.2036 & 0.0073 & 0.0075 & 0.0016 & 0.0039 & 0.0008 & 0.0045 & 0.0050 \\
\hline Max. & 0.3014 & 0.0123 & 0.0421 & 0.0027 & 0.0202 & 0.0066 & 0.0104 & 0.0392 \\
\hline Min. & 0.0613 & 0.0047 & 0.0000 & 0.0004 & 0.0005 & 0.0000 & 0.0000 & 0.0000 \\
\hline SD & 0.0287 & 0.0010 & 0.0125 & 0.0005 & 0.0027 & 0.0018 & 0.0024 & 0.0052 \\
\hline RSD & $14 \%$ & $14 \%$ & $166 \%$ & $29 \%$ & $70 \%$ & $216 \%$ & $52 \%$ & $103 \%$ \\
\hline MRD & $70 \%$ & $69 \%$ & $460 \%$ & $76 \%$ & $419 \%$ & $716 \%$ & $128 \%$ & $682 \%$ \\
\hline
\end{tabular}

\begin{tabular}{|l|l|l|l|l|l|l|l|l|}
\hline Specie & Mn & Na & Nb & Ni & Sr & Sn & PO4 & SO4 \\
\hline
\end{tabular}




\begin{tabular}{|l|c|c|c|c|c|c|c|c|}
\hline Avg. & 0.0002 & 0.0211 & 0.0005 & 0.0005 & 0.0021 & 0.0016 & 0.0046 & 0.0091 \\
\hline Max. & 0.0012 & 0.0519 & 0.0148 & 0.0009 & 0.0032 & 0.0026 & 0.1346 & 0.0319 \\
\hline Min. & 0.0000 & 0.0000 & 0.0000 & 0.0001 & 0.0002 & 0.0008 & 0.0002 & 0.0004 \\
\hline SD & 0.0003 & 0.0097 & 0.0022 & 0.0002 & 0.0004 & 0.0003 & 0.0139 & 0.0079 \\
\hline RSD & $131 \%$ & $46 \%$ & $419 \%$ & $45 \%$ & $18 \%$ & $16 \%$ & $305 \%$ & $87 \%$ \\
\hline MRD & $438 \%$ & $146 \%$ & $2729 \%$ & $96 \%$ & $91 \%$ & $58 \%$ & $2841 \%$ & $251 \%$ \\
\hline
\end{tabular}



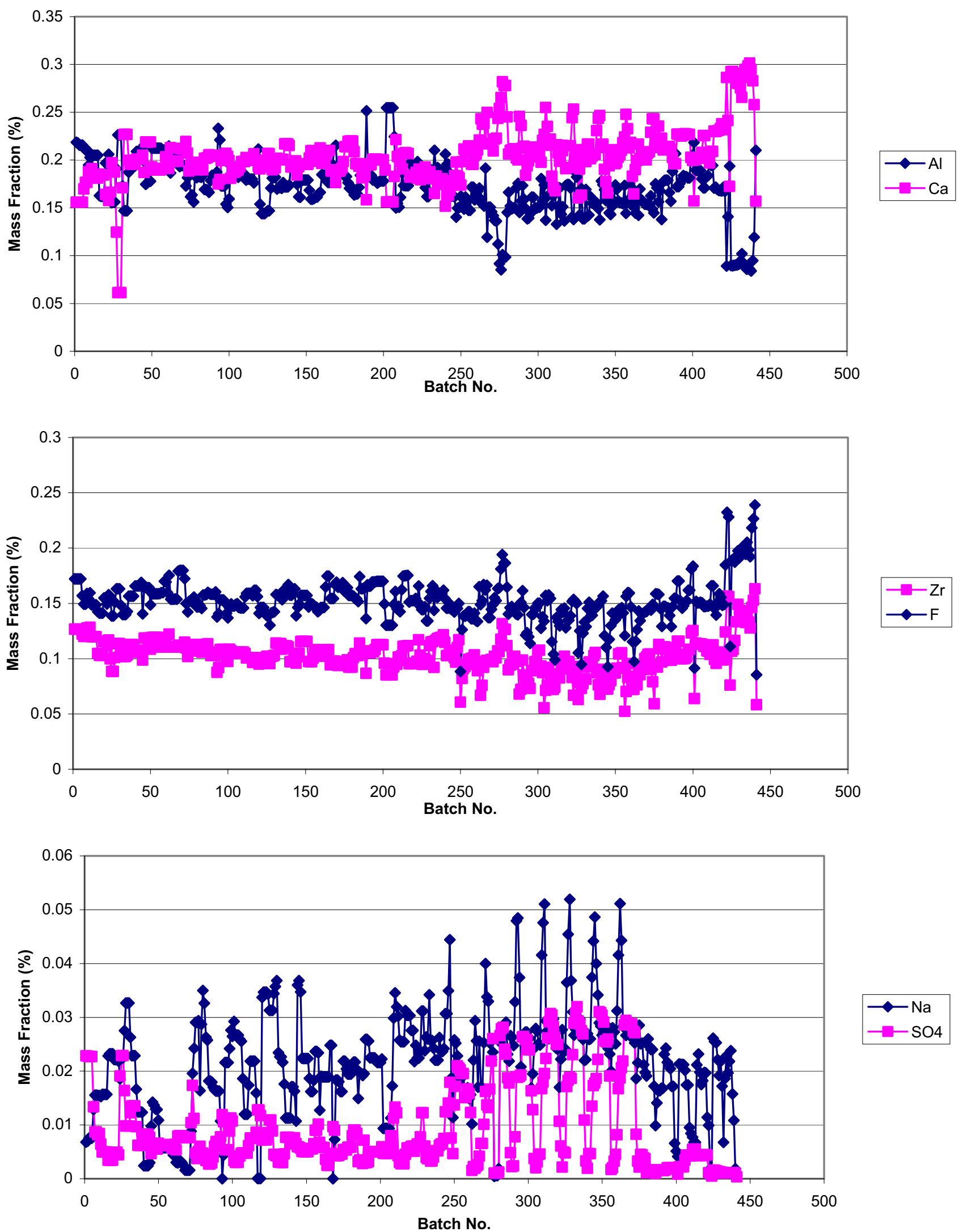

Figure 4-5: Batch-by-batch composition variation (major species, Phase 3 study-Case A). 

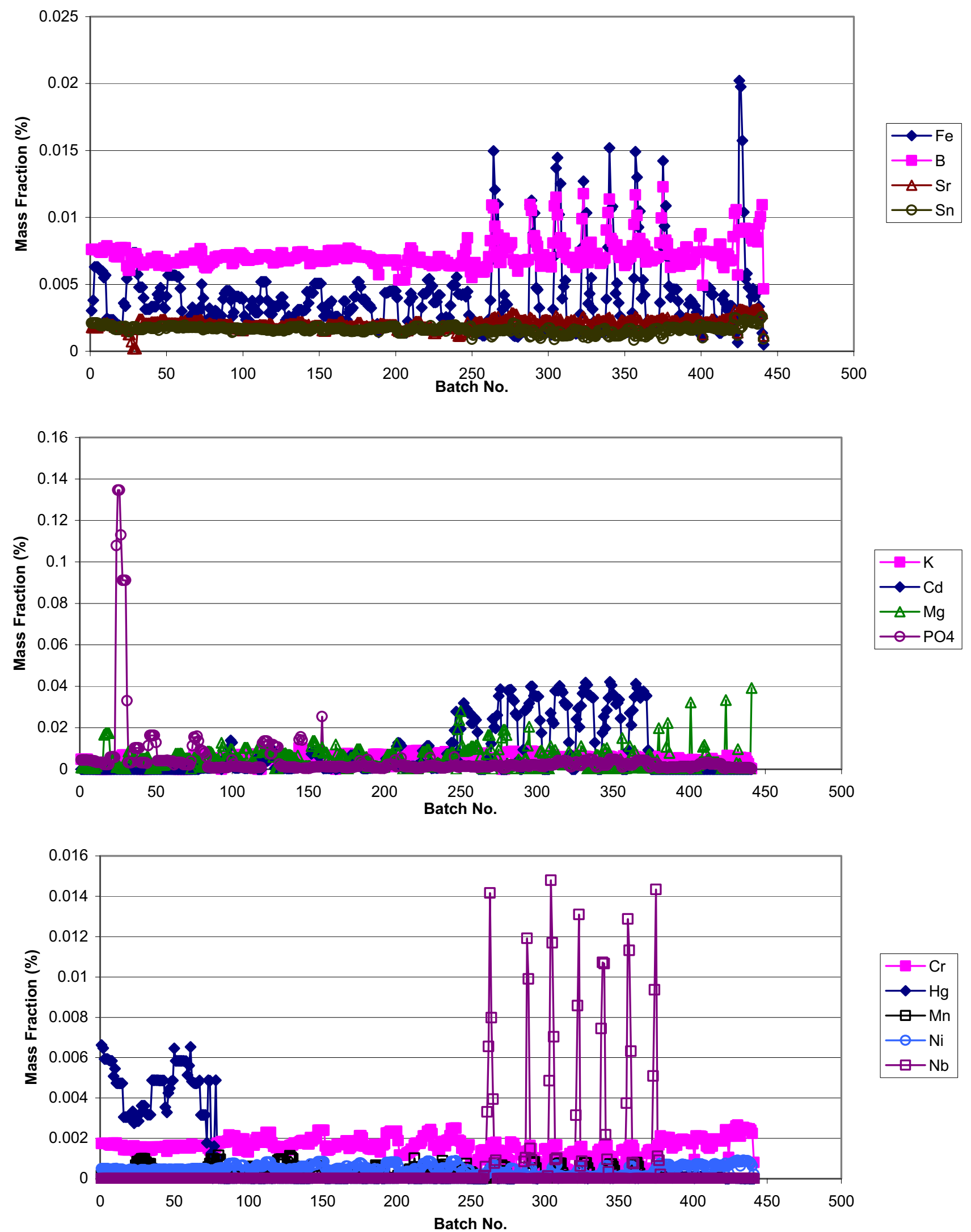

Figure 4-6: Batch-by-batch composition variation (major species, Phase 3 study-Case A). 
Table 4-6 indicates RSDs of $17 \%, 15 \%$, and $12 \%$ for Al, Zr, and F, respectively for the Case A of the Phase 3 study. These numbers represent a factor of 2-3 reduction in the RSDs of composition from what is likely without optimized retrieval (see Section 1.2). The reduction is not as dramatic as for the Phase 1 and 2 studies, but is still significant. The MRDs for this case were $51 \%, 59 \%$, and $57 \%$ for $\mathrm{Al}, \mathrm{Zr}$, and $\mathrm{F}$, respectively. These also are lower by factors of 1.4-2.7 than the corresponding values of $138 \%, 81 \%$, and $84 \%$, respectively from the non-optimized case.

Reference to Figure 4-5 suggests that removal of a relatively small number of outlier batches may reduce the RSD even more. This was done and the results are summarized in Table 4-8. Removal of those batches having a fractional deviation of $34 \%$ or more from the average composition for $\mathrm{Al}, \mathrm{Zr}$, or $\mathrm{F}$ (about $10 \%$ of all batches) reduced the RSDs by another $30-40 \%$ for $\mathrm{Al}, \mathrm{Zr}$, and $\mathrm{F}$ to $12 \%, 12 \%$, and $8 \%$, respectively, and reduced the MRDs to $32 \%, 34 \%$, and $31 \%$. This is further discussed in Section 4.3.

Table 4-8: Statistics of composition variation of optimized species (Phase 3 study-Case A, with outliers removed).

\begin{tabular}{|l|c|c|c|}
\hline Specie & Al & Zr & F \\
\hline Avg. & 0.1754 & 0.1023 & 0.1512 \\
\hline Max. & 0.2261 & 0.1284 & 0.1846 \\
\hline Min. & 0.1192 & 0.0677 & 0.1040 \\
\hline SD & 0.0207 & 0.0118 & 0.0127 \\
\hline RSD & $12 \%$ & $12 \%$ & $8 \%$ \\
\hline MRD & $32 \%$ & $34 \%$ & $31 \%$ \\
\hline
\end{tabular}

Case B (optimized retrieval with mixed calcine layers as input to Monte Carlo model):

Table 4-9: Statistics of composition variation of optimized species (Phase 3 study-Case B).

\begin{tabular}{|l|c|c|c|}
\hline Specie & Al & Zr & F \\
\hline Avg. & 0.1724 & 0.1028 & 0.1526 \\
\hline Max. & 0.2835 & 0.1525 & 0.2250 \\
\hline Min. & 0.0860 & 0.0408 & 0.0555 \\
\hline SD & 0.0320 & 0.0173 & 0.0215 \\
\hline RSD & $19 \%$ & $17 \%$ & $14 \%$ \\
\hline MRD & $64 \%$ & $60 \%$ & $64 \%$ \\
\hline
\end{tabular}



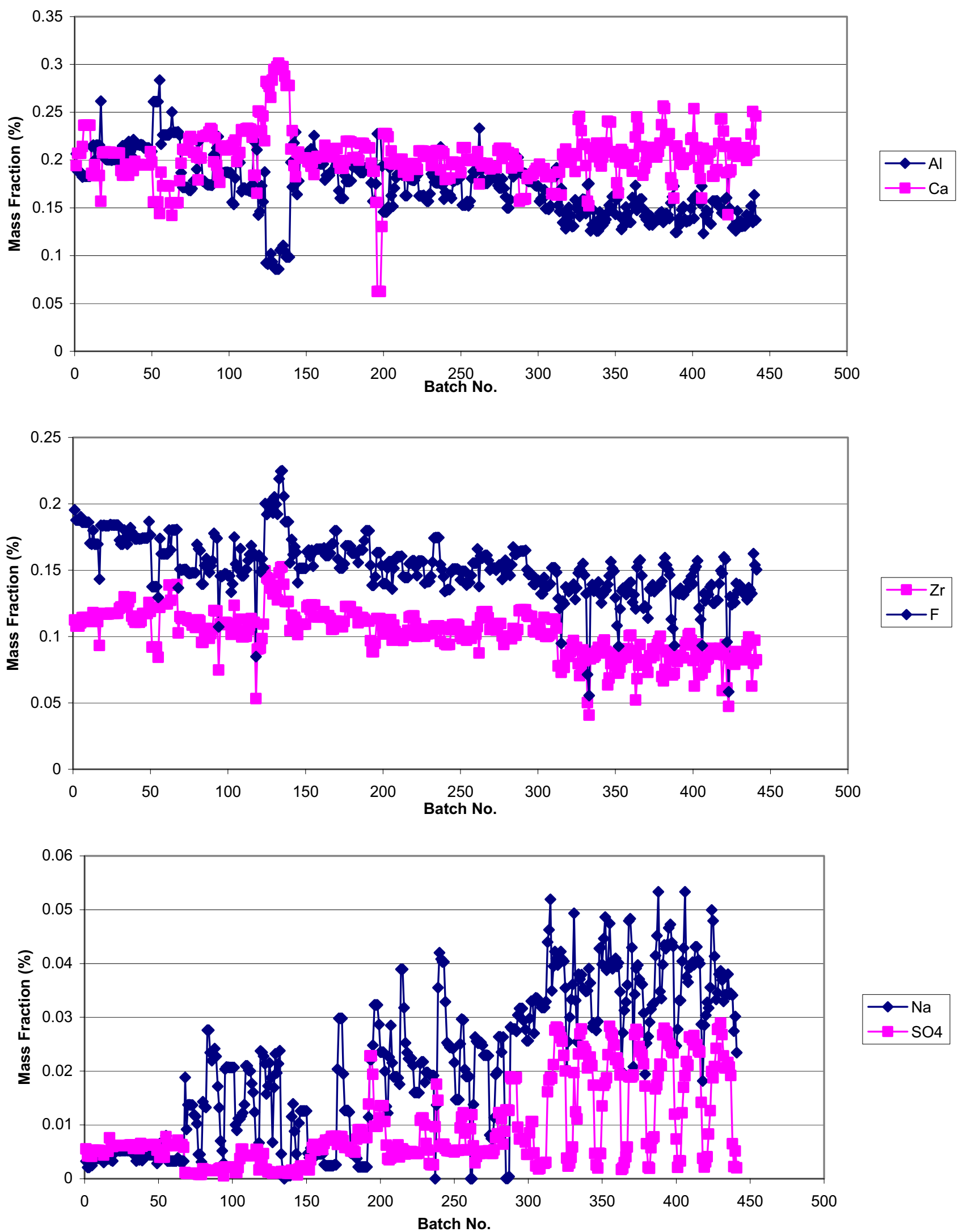

Figure 4-7: Batch-by-batch composition variation (major species, Phase 3 study-Case B). 
Table 4-9 indicates RSDs of $19 \%, 17 \%$, and $14 \%$ for $\mathrm{Al}, \mathrm{Zr}$, and $\mathrm{F}$, respectively for Case $\mathrm{B}$ of the Phase 3 study. Taken at face value these values suggest that mixing of different calcines upstream of the blending bin increases slightly the composition variation after optimization of the third retrieval discussed in Section 3.2. However, the increase is slight. In addition, the 5-ft mixing zone length is probably a significant overestimate and the impact of a more reasonable estimate for this parameter may show little or no effect of mixing. However, in light of the unexpected result that the Phase 2 simulation showed smaller composition variation than Phase 1 (see Section 4.2.2.2), the indicated increase in composition variation with the mixing model may be misleading. This is discussed further in Section 4.3.

Case $\mathbf{C}$ (retrieval of blended calcine per recipe generated with unmixed calcine layers):

Table 4-10: Statistics of composition variation of optimized species (Phase 3 study-Case C).

\begin{tabular}{|l|c|c|c|}
\hline Specie & Al & Zr & F \\
\hline Avg. & 0.1724 & 0.1026 & 0.1522 \\
\hline Max. & 0.2737 & 0.1598 & 0.2342 \\
\hline Min. & 0.0840 & 0.0570 & 0.0912 \\
\hline SD & 0.0289 & 0.0152 & 0.0184 \\
\hline RSD & $17 \%$ & $15 \%$ & $12 \%$ \\
\hline MRD & $59 \%$ & $56 \%$ & $54 \%$ \\
\hline
\end{tabular}



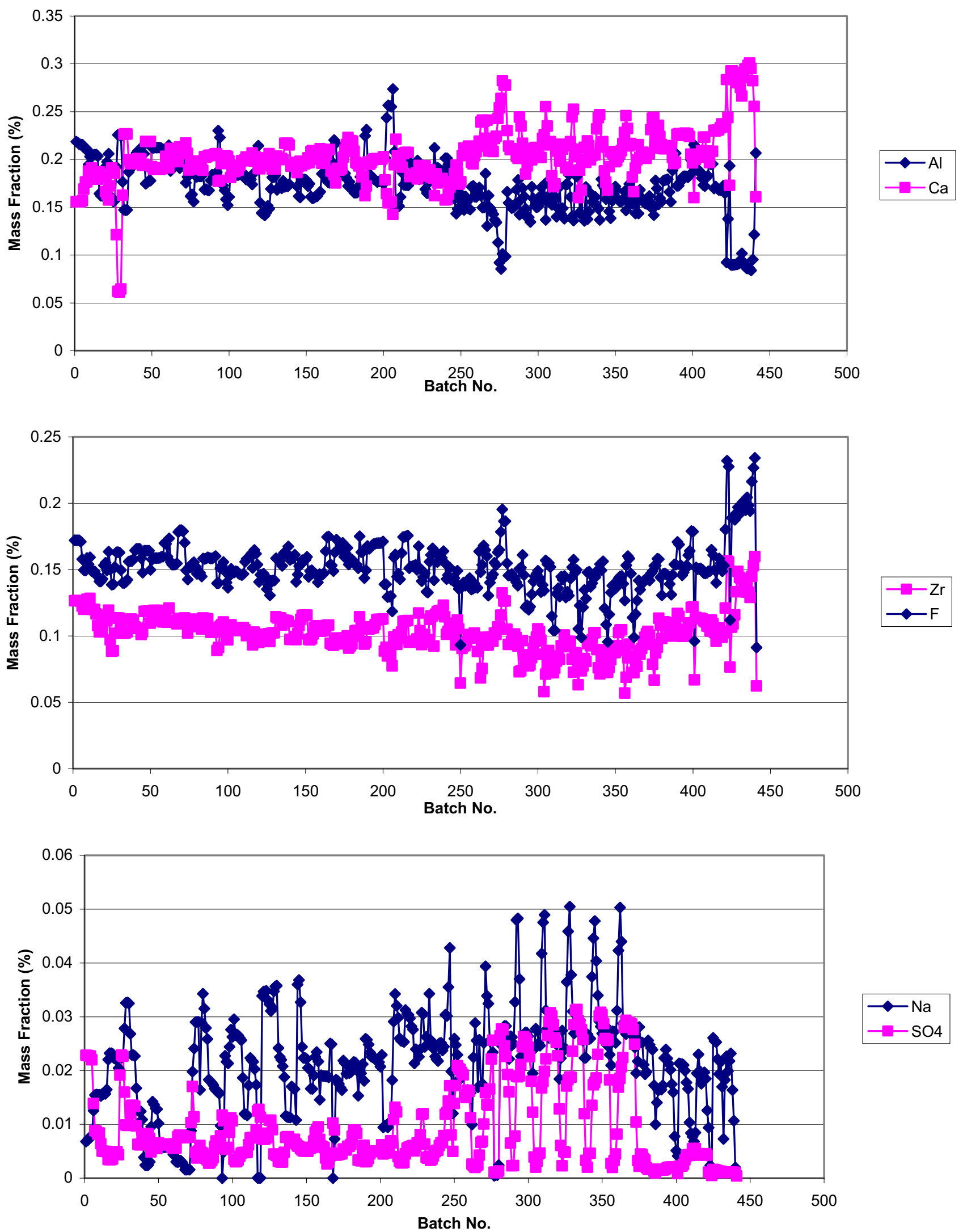

Figure 4-8: Batch-by-batch composition variation (major species, Phase 3 study-Case C) 
Table 4-10 indicates RSDs of $17 \%, 15 \%$, and $12 \%$ for $\mathrm{Al}, \mathrm{Zr}$, and $\mathrm{F}$, respectively for Case $\mathrm{C}$ of the Phase 3 study, just slightly lower than the values for Case A. Again, taken at face value these values suggest that optimization of retrieval based only on the nominal compositions of the nonmixed portions of the calcine strata in the bins should be provide good results, without any account being taken of mixing in the optimizing process. The use of a liberal value of $5 \mathrm{ft}$ for the mixing zone length further supports this conclusion. However, as in the Case A-Case B comparison it may be questionable whether much significance may be attached to the differences between the Case $\mathrm{C}$ and the Case A RSDs.

\subsection{Discussion}

The results of the Phase 1 study show that large reduction in the composition variation of blended calcine is theoretically achievable with optimized retrieval. These results provide a strong incentive to consider retrieval optimization as an alternative to qualifying the calcine treatment process (and resulting waste forms) for widely varying compositions in the calcine fed to the process. However, it must be borne in mind that none of the retrieval systems considered in studies to date allowed for withdrawal of calcine from any bin in any binset at any time, as postulated for the Phase 1 study. Based on comments made in the Ref. 6, practical considerations of worker safety, operational time required to move suction nozzles from bin to bin, and capital costs, the hypothetical retrieval system addressed in the Phase 1 study is probably not feasible. Because of space restrictions and equipment costs, only a limited number of bins will likely be available to supply calcine to the blending bin at any given time. Reference 6 suggest that this number is most likely either two or six.

The results of the Phase 2 study shows that restriction of the number of suction nozzles to six during retrieval may result in little degradation in the results achievable with the more complex system treated in Phase 1. Indeed, the computational results presented here suggest that better results might be achieved with the 6-nozzle system. Though this thought is appealing, a little reflection clearly shows it to be false. The universe of possible retrieval sequences available in the Phase 1 study includes all those available in the Phase 2 study, and many, many more in addition. With more retrieval sequences (i.e., recipes) to choose from the absolute best recipe available within the Phase 1 study constraints must therefore be at least as good as the best recipe within the Phase 2 constraints (and probably better). Therefore, it is concluded that (a) the "best" recipe obtained from the Phase 1 simulation is indeed not the absolute best achievable, (b) if we searched longer during the Phase 1 calculations we would have eventually found the Phase 2 recipe, and (c) if we had searched longer still, we may have found an even better one! In other words, as suggested by the remarks in Section 4.1.1, the Monte Carlo scheme used here simply cannot sample a large enough fraction of the total universe of possible recipes to find "the very best ones".

The now-obvious conclusion that the recipes achieved in the simulations were not true optima casts some doubt on the tentative conclusions reached in the Phase $3 \mathrm{~B}$ and $3 \mathrm{C}$ studies, relative to the impact of mixing on the optimized results. The comparatively small differences between the cases with and without mixing may be attributable to the fact that better approximations to the optimum were achieved in some cases than in others. However, while the results do not prove that mixing has a small effect, they are at least consistent with this possibility.

We emphasize that, in spite of the foregoing remarks regarding the approximate nature of the optima that were achieved, the Monte Carlo scheme still produced recipes which are "very good" in an absolute sense, when compared with the non-optimized recipe discussed in Section 1.2. It should also be kept in mind that, to the extent the calcine database used is accurate, the blended calcine compositions described here would actually have been achieved using the postulated retrieval systems 
with the recipes that were derived. Thus, the fact that true optima were not achieved in the simulations does not detract from the usefulness of the results.

In particular the relatively low composition variations indicated in the Phase 2 study could likely be realized with a system similar to those considered in Ref. 6 . The same statement applies to the Phase 3 study with only two suction lines in the retrieval system, though in this case the variation in composition of the blended, retrieved calcine is greater. However, the fact that we didn't actually achieve a true optimum now may work in our favor. That is, we might reasonably expect to improve the 2-suction recipe by performing more Monte Carlo simulations or by building some intelligence into the searching scheme as was suggested in Section 4.1.1.

Another alternative may be suggested by Figure 4-5. The "spikes" in the composition plots suggest that perhaps no recipe can be found with only two suction lines that completely eliminates the outlying spikes in composition. However, as suggested by remarks in Section 4.2.3.2, by "throwing out" about $10 \%$ of the blended batches (i.e., the outliers) the RSD of the remaining blended calcine compositions can be reduced from about $17 \%$ to about $12 \%$. While at this point it is not clear how many waste forms would need to be qualified to cover the range of compositions indicated by the results presented here, it may be that a single waste form could accommodate a $12 \%$ variation with an acceptably-high waste loading. If this were true, then the $10 \%$ of the batches that were "thrown out" might be processable with the same recipe at a lower waste loading. Because only $10 \%$ of the batches are outliers, this would not significantly impact the total volume of the final waste form. However, if only a single recipe had to be qualfied for disposal in the repository, considerable cost savings might be realized.

Assuming that glass is selected as the final waste form, the results provided here would have to be reviewed by glass formulation experts to determine if optimized retrieval and blending of calcine would significantly reduce the burden of qualifying the final waste forms. This determination would require detailed data on waste form qualification costs and retrieval economics in order to intelligently minimize the total cost of retrieving and processing/shipping the calcine. This type of determination was beyond the scope of the present study.

Finally, we note that the recent National Research Council review of the INEEL High Level Waste Program (Ref. 7) recommended sampling and characterization of stored calcines prior to deciding on a preferred option for treatment and disposal. The current studies suggest a possible path forward in this regard. A limited sampling effort could first be performed. The objectives of this effort would be to (a) quantify the quality of the data in the calcine database used in these studies (Ref. 1), and (b) obtain a sufficient number of calcine samples (perhaps as few as 5-10) to bracket the spectrum of calcine types in the bins (in some sense, yet to be determined). These samples would be completely characterized with respect to composition and radionuclide content. They (or cold surrogates based on the complete characterization of the samples) would also be used in treatment studies to determine the sensitivity of key unit operations (e.g., direct vitrification, TRUEX separations, etc.) to variations in calcine feed composition. Then, using the quantitative measures of quality of the calcine database and the process sensitivity data, additional retrieval optimization studies would be performed to determine whether the likely calcine composition variations that an optimized retrieval system would deliver could be accommodated by the treatment process. If so, then additional sampling would not be required and the considerable expense of sampling all calcines in all bins would not be incurred. If, on the other hand, these efforts show that the treatment process will not accommodate feed variations with optimized retrieval then there would be clear justification for directing funds away from optimized retrieval and toward detailed calcine sampling and characterization. 


\section{CONCLUSIONS \& RECOMMENDATIONS}

This study was directed at determining the degree of homogeneity that can theoretically be achieved in the composition of retrieved calcine by optimizing the sequencing of retrieval from the binsets and blending the retrieved calcine in a reasonably-sized blending bin. The results show that optimized retrieval can provide a significant reduction in the variation of calcine composition fed to a downstream treatment process. The study produced a number of useful conclusions and recommendations as outlined below.

\subsection{Conclusions}

6. Fully optimized retrieval and blending may result in sufficient homogeneity to justify a single flowsheet for calcine treatment.

7. The retrieval system required to achieve fully optimized calcine retrieval is probably prohibitively costly and complex.

8. A partially optimized retrieval system employing one retrieval line for every binset (similar to one that has been studied and costed in an earlier INEEL study) can reduce the variations in $\mathrm{Al}$, $\mathrm{Zr}$, and $\mathrm{F}$ concentrations in retrieved calcine to at least the level that was achieved in the study with a fully optimized retrieval system described in item 6 . Relative standard deviations for $\mathrm{Al}, \mathrm{Zr}$, and $\mathrm{F}$ of less than $2 \%$ were achieved; corresponding maximum relative deviations for these species were below $10 \%$.

9. Approximate optimization of a minimum-cost retrieval system, employing only two suction lines which are moved from one bin to another after bins are fully drained, did not achieve a near-uniform composition for all batches - a fraction of the batches for this case had large deviations from the average composition. However, composition variations with this system are still a factor of 2-3 lower than with a non-optimized system.

10. The study revealed no strong effect of mixing between strata in the bins on the homogeneity of the blended calcine batches. The results suggests that there is little effect of moderate mixing for the batch and chunk sizes assumed. However, the mixing model incorporated in this study is somewhat simplified and requires further investigation.

\subsection{Recommendations}

10. Direct efforts at defining a rationale and a strategy for sampling calcine in the binsets. This effort should be based on empirical determinations of robustness of the candidate treatment processes to feed variations, studies of the effects on key treatment operations of all elemental species that may be present in the calcines, and the degree of homogenization that can be achieved during calcine retrieval. (One possible strategy has been suggested in Section 4.3 of this report.)

11. Perform optimized retrieval studies on the effect of uncertainty in the locations of the interfaces between calcine strata in the bins.

(In actual practice the retrieval process would be controlled by real-time assay of the calcine being retrieved. This is necessary because estimated calcine volumes from Ref. 1 used in optimizing the retrieval recipe are not exact. Thus, "blind" retrieval, based solely on advance 
calculations of an optimal retrieval sequence, would likely not produce the desired degree of homogeneity of the retrieved calcine.)

12. Develop real-time assay techniques for use during retrieval to identify interfaces between calcine types. Such techniques would be required for optimized retrieval and would provide the information needed to control the retrieval process, as discussed in item 11, above.

13. Perform additional retrieval simulations that allow variation of

(a) the number of calcine portions that make up a blended batch of calcine

(b) the size of the blending bin

(c) non-fixed "chunk" sizes during retrieval from different bins

(d) the number of trial recipes and the recipe selection strategy used in the Monte Carlo scheme

(e) the mixing length parameter in the mixing model

to determine whether additional reductions in composition variations of the blended calcine might be achieved.

14. Plan future glass composition variation studies (CVSs) to reflect expected calcine feed variations described in this report.

(In particular, further studies are needed to determine if these variations can be effectively handled by adjusting waste loading in the glass.)

15. Perform a tradeoff study to assess the merit (from a cost standpoint) of optimizing the retrieval system vs qualifying multiple flowsheets and/or final waste forms to accommodate large variations in calcine feed composition.

16. Review ITDF functional requirements and design to account for the specifics of likely approaches to sampling, retrieving and blending calcine.

17. The study results are useful only to the extent that the calcine composition data which were used are reliable. Thus, at a minimum it is essential that a limited number of random calcine samples be extracted from the bins to qualify the database. Sampling may also be necessary to characterize the degree of mixing that has occurred in the bins. Thus, some of the capabilities and space requirements of the ITDF must accommodate development work for bin sampling methods and calcine characterization. This underscores the need to provide mockup and hotcell capabilities in the ITDF.

18. The upper practical limit on the size of a calcine blending bin should be determined. Again, this would presumably be done in the ITDF. 


\section{REFERENCES}

1. Staiger, M. D., Calcine Waste Storage at the Idaho Nuclear Technology and Engineering Center, INEEL/EXT-98-00455, June 1999.

2. Personal communications between B. H. O’Brien and L.O. Nelson, February, 2000.

3. B. H. O'Brien, Estimation of Alkali Metal Mole Percent and Weight of Calcined Solids for ICPP Calcine, INEL-95/0184, March 1995.

4. Raytheon Engineers and Constructors, Inc., Idaho Chemical Processing Plant Bin Set 1 Calcine Recovery Project: Phase I and II Special Studies Report Volume I, DE-AC07-89ID12679, February 1995.

5. Fluor-Daniels, Inc. (Government Services Operating Company), Idaho Chemical Processing Plant Waste Treatment Facilities Feasibility Study, Delivery Order 94-36, 30\% Design Review Package, June 1997, 60\% Design Review Package, August 1997, and 90\% Design Review, October 1997.

6. Gifford, S.E., Calcine Retrieval and Transportation, Engineering Design File for Project File Number O2BD7 [Project/Task: Waste Treatment Facility Study, Subtask: Retrieve calcine from CSSFs and deliver it to the Waste Treatment Facility], Function File Number - SPRWTS-01, EDF Serial Number - EDF-WTS-002, February 4, 1998.

7. $\quad$ Alternative High-Level Waste Treatments at the Idaho National Engineering and Environmental Laboratory, National Research Council, National Academy Press, Washington, DC, 1999. 